\title{
Tecnología de arponaje en la costa del desierto de Atacama, norte de Chile
}

\section{Harpoon technology in the Atacama desert coast, northern Chile}

\author{
Benjamín Ballester R. ${ }^{1}$
}

\begin{abstract}
Resumen
La caza marina fue una actividad esencial en el modo de vida de los habitantes del litoral del desierto de Atacama, al norte de Chile. Un pilar de su estructura social que tenía como base material los medios técnicos para realizarla, como las embarcaciones y su sofisticado dispositivo de arponaje, descansando en este último la posibilidad de capturar y arrastrar hacia tierra firme presas que superaban sustancialmente el tamańo y peso del cazador. En la actualidad muy poco se sabe del arponaje desde la arqueología. Por esto ahondaremos en su morfología, composición, cualidades técnicas, diversidad instrumental y cronología a partir de una muestra de 129 cabezales de arpón de distintos períodos de la prehistoria (6500 cal AP a momentos coloniales). Comenzaremos definiendo conceptualmente la función del arpón, seguido de un primer ejercicio tipológico que ordene la multiplicidad de formas y soluciones a nivel mundial para ubicar los ejemplares analizados. En un segundo ejercicio tipológico clasificaremos la diversidad de expresiones locales, diferenciándolas materialmente y situándolas en la secuencia cronológica. Solo después de esta necesaria etapa clasificatoria podremos aproximarnos a su tecnología de arponaje, el rol de los cazadores como sujetos sociales y el papel de la caza en la sociedad litoral.
\end{abstract}

Palabras claves: arponaje, caza marina, conocimiento, organización social, tecnología.

\begin{abstract}
Marine hunting was an essential activity in the way of life of the Atacama Desert littoral inhabitants, at northern Chile. A pillar in their social structure that was based in the material means to realize it, as boats and their sophisticated harpoon device, resting in the last the possibility of capture and drag mainland preys that substantially outweigh the hunter. Currently the knowledge of harpooning is scarce in archaeology. For this reason we will delve in their morphology, composition, technical qualities, instrumental diversity, and chronology from a 129 harpoon heads sample from different prehistoric periods (6500 cal BP to colony). We will begin conceptually defining the harpoon function, followed by a first typological exercise to order the multiplicity of forms and solutions in a global scale to place the analyzed specimens. In a second typological exercise we will classify the local expression diversity, differentiating them materially and placing them chronologically. Only after this necessary classificatory stage we can approach to their harpoon technology, the hunter role as a social subject, and the position of hunting in the littoral society.
\end{abstract}

Keywords: harpooning, marine hunting, knowledge, social organization, technology.

Recibido: 18 octubre 2016. Aceptado: 29 marzo 2017.

1 UMR 7041 ArScAN - Équipe Ethnologie Préhistorique - Université Paris 1 Panthéon Sorbonne. FRANCIA. Email: benjaminballesterr@gmail.com 


\section{Introducción}

Apenas arribaron los primeros europeos a las áridas costas del desierto de Atacama en la segunda mitad del siglo XVI, no tardaron en hallar a los nativos locales. Se trataba de comunidades de cazadores, pescadores y recolectores marinos que por su rudimentario modo de vida en comparación al resto de los pueblos andinos de agricultores y pastores, fueron descritos como personajes barbáricos y salvajes, caricaturizados como desnudos bebedores de sangre de lobo marino que no servían a nadie y se sustentaban solo de pescado crudo y mariscos (Casassas, 1992; Lizárraga, 1999 [1603-1609]; Pernaud, 1990; Sayago, 1973 [1874]).

Pero aun por sobre esta imagen cargada de prejuicios etnocentristas y evolucionistas, los navegantes se sorprendieron de la destreza de este pueblo en la navegación sobre frágiles embarcaciones hechas de flotadores de piel de lobo marino, su capacidad de buceo, su economía excedentaria y apertura al intercambio, pero por sobre todo de su habilidad única para pescar y cazar en alta mar (Bethune, 1848 [1622]; Bibar, 1966 [1558]; Bollaert, 1854, 1860; Feuillée, 1714; Pernaud, 1990; Pretty, 1904 [1599]; Vaux, 1854; Vázquz de Espinoza, 1948 [1630]). La caza marina integraba todas estas aptitudes en hazañas llenas de braveza y valentía para capturar algunos de los animales más grandes e imponentes de la región, faenas que solo eran posibles gracias a los artefactos más sofisticados de su universo material, balsas y arpones. Según relata el fray Reginaldo de Lizárraga (1999 [1603-1609], p. 378) en los primeros años del siglo XVII, para la caza de atunes los balseros "no andan en cuadrillas como en España, sino de uno en uno; sale el indio pescador en busca de él, dos y más leguas á la mar con su balsilla de cuero de lobos; lleva su arpón, físgale, dale soga hasta que se desangra; desangrado le saca á la costa”.

Pocos años más tarde otro clérigo español, Antonio Vázquez de Espinoza (1948 [1630], pp. 618-619), registraba una de las escenas más conocidas e importantes sobre la caza marina en la región:

...hay en aquella provincia cantidad de cobre, del cual hacen unas púas, o garrochuelas menores, que garrochones, estos los ponen en unas astas pequeñas de tal suerte dispuestas, y atadas con un látigo de cuero de lobo a la muñeca, van a tirar a las ballenas: las cuales de ordinario en aquella costa duermen de medio día para arriba, dos, o tres horas con gran reposo, y profundo sueño, sobre aguadas, y con una ala pequeńa, que tienen sobre el corazón se cubren la cabeza para dormir por el sol. Entonces que la a asechado el indio cuando duerme, en que esta diestro, llega en su balsilla de lobo, en que va para valerse de ella sin que la pueda perder, y se llega donde la ballena duerme: y le da un arponazo debajo del ala, donde tiene el corazón, e instantáneamente se deja caer al agua, por escaparse del golpe de la ballena; que viéndose herida se embravece dando grandes bramidos, y golpes en el agua, que la arroja muy alta con la furia, y cólera que le causa el dolor, y luego tira bramando hacia la mar, basta que se siente cansada, y mortal; en el intertanto el indio vuelve a cobrar su babilla, y se viene a tierra a ojear, y atalayar adonde viene a morir a la costa, y así están en centinela, hasta que la ven parar.

En mayo de 1710 el mercader francés Louis Feuillée (1714, pp. 590-591; la traducción es nuestra) examinó de cerca y montó una de estas balsas en la bahía de Cobija. Al describirla relata que sobre ella los balseros "posan todas sus provisiones, que consisten en una gran calabaza repleta de agua, y sus armas, que son un arco, flechas y un especie de dardo para varar los peces grandes", y que es para "alejar o para defenderse de contra estos peces que los indios embarcan siempre con ellos un gran dardo provisto de una punta en su extremidad".

Los relatos escritos y gráficos (Figura 1) de los primeros siglos de contacto son certeros sobre la capacidad de caza marina de los nativos del litoral desértico. Más aún, nos entregan valiosas pistas sobre su tecnología, estrategias y organización social en las faenas de caza marina. Detallan sintéticamente, pero sin pasar por alto sus dispositivos de caza, resaltando en las escenas los arpones, sus formas, materiales y composición. Lamentablemente la ar- 


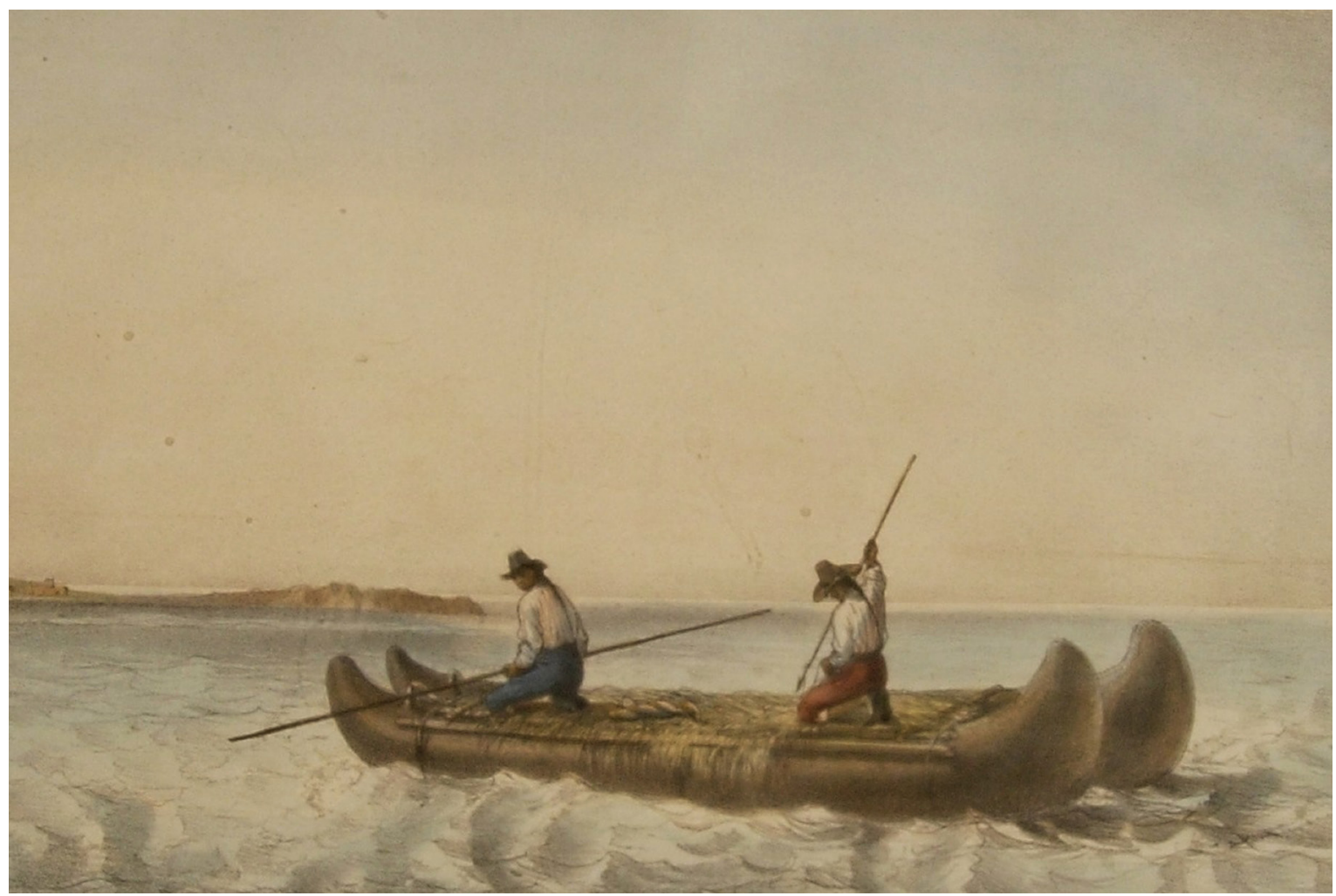

Figura 1. Representación de dos balseros en faenas de caza sobre una balsa de cuero de lobo marino frente a las costas de Cobija en 1830, aquel de popa porta un arpón en sus manos (D’Orbigny, 1847).

queología poco ha ahondado en el estudio de esta tecnología, dejando un gran vacío sobre el papel de la caza marina en tiempos prehispánicos (Llagostera, 1989). Enorme paradoja si consideramos las elocuentes representaciones del estilo de arte rupestre de El Médano, una expresión gráfica sobre la roca que retrata con pigmento rojo cientos de escenas donde embarcaciones capturan con sogas presas marinas como ballenas, delfines, tortugas, tiburones y peces espadas (Ballester, 2018; Ballester y Álvarez, 2014-2015; Ballester y Gallardo, 2016; Berenguer, 2009; Contreras et al., 2008b; Gallardo et al., 2012; Mostny y Niemeyer, 1983, 1984; Niemeyer, 2010; Núñez y Contreras, 2003, 2006, 2008).

Paralelamente otras evidencias arqueológicas han abierto el debate sobre el papel de la caza marina y navegación. Por una parte, la presencia en sitios basurales de restos de animales marinos que por su tamaño y hábitat debieron capturarse utilizando la tecnología conjunta de embarcaciones y arponaje, con fechas tan antiguas como los $7000 \mathrm{cal}$ AP y prolongándose hasta tiempos de contacto europeo (Báerez et al., 2016; Ballester et al., 2017; Bird, 1943; Bittmann, 1978; Castro et al., 2012; Contreras et al., 2011; Llagostera, 1990; Mostny, 1942; Núñez et al., 1974; Núñez, 1974; Olguín et al., 2014, 2015; Rebolledo et al., 2015; Schiappacasse y Niemeyer, 1984). Por otra, las transformaciones en su patrón de asentamiento, sistema de movilidad y economía han demostrado el papel protagónico de la navegación a partir de este mismo momento y a lo largo de la secuencia posterior, donde una de sus funciones principales fue acceder a presas marinas de mayor tamaño en alta mar (Ballester y Gallardo, 2011; Ballester et al., 2017; Bittmann, 1978; True, 1975).

Sin embargo los arpones han sido invisibilizados de la prehistoria a pesar de que su presencia como ofrenda en contextos fúnebres es sumamente común al menos desde los $6500 \mathrm{cal} \mathrm{AP} \mathrm{y} \mathrm{hasta} \mathrm{la} \mathrm{llegada} \mathrm{del}$ 
mundo europeo, de igual manera que sus secciones como barbas óseas, cabezales líticos y fragmentos de astiles de arpón en los depósitos basurales de los sitios habitacionales (Ballester et al., 2014a, 2014b, 2017; Barraza, 1981; Bird, 1943; Bittmann, 1984; Boisset et al., 1969; Castelleti, 2007; Contreras et al., 2008a, 2011; Latcham, 1910; Llagostera, 1989; Mostny, 1964; Núñez et al., 1974; Salazar et al., 2015; Silva y Bahamondes, 1969; Spahni 1967). ¿Las razones? Tal vez las miradas, porque el registro yace a la espera.

Con la necesidad de llenar estos vacíos arqueológicos presentamos un primer acercamiento a la tecnología de arponaje del litoral desértico de Antofagasta a partir del análisis de una colección de 129 cabezales de arpón de distintos momentos de la prehistoria, complementándolo además con la evidencia de arpones completos, fragmentos en sitios habitacionales y restos de fauna marina. Comenzaremos revisando el papel de la caza marina a lo largo de las costas americanas para tener una visión comparativa sobre la práctica tanto desde una variable tecnológica como económica, política y simbólica. Desde aquí podremos definir conceptualmente la función del arpón y realizar un primer ejercicio tipológico que ordene la multiplicidad de formas y soluciones a nivel mundial para ubicar los ejemplares analizados. En un segundo ejercicio tipológico clasificaremos la diversidad de expresiones del desierto de Atacama, diferenciando materialmente los tipos según criterios técnicos y situándolos en la secuencia cronológica. Solo después de esta necesaria etapa clasificatoria y en conjunto con información complementaria de la prehistoria local, podremos aproximarnos a la tecnología de arponaje, el rol de los cazadores como sujetos sociales y el papel de la caza marina en la sociedad litoral del desierto de Antofagasta.

\section{Las sociedades americanas cazadoras de grandes presas marinas}

La etnografía, arqueología e historia se han encargado de mostrar que la caza de grandes presas marinas fue común entre ciertas sociedades cazadoras recolectoras que habitaron las costas del continente americano. Aún más, han puesto en evidencia que en algunas de ellas esta práctica adquirió un rol protagónico en su modo de vida, no solo por el beneficio alimentario que generaban para su supervivencia física los enormes volúmenes de comida, sino también como pilar en sus procesos de construcción sociocultural al convertir a estos animales en agentes mitológicos activos y referentes representacionales de su universo simbólico e imaginario social (Acosta, 1590; Bockstoce, 1976; Caulfield, 1993; Chapman, 2012; Coté, 2010; Krupnik, 1987; Krupnik y Kan, 1993; Lantis, 1938; Larsen y Rainey, 1948; Leroi-Gourhan, 1935; Mulville, 2002; Paton y Savelle, 2006; Porcasi y Fujita, 2000; Quiroz, 2015; Reeves, 2002; Waterman, 1920).

Quizás el caso etnográfico mejor documentado proviene de los esquimales del Polo Norte. Marcel Mauss (1906) los definió tempranamente como un pueblo esencialmente costero que albergaba en su seno a expertos cazadores de cetáceos, focas, narvales y morsas en sus kayaks, utilizando arpones con sistema de boyas o flotadores hechos de pieles de foca (Birket-Smith, 1953; Boas, 1888, 1907; Heizer, 1943; Larsen y Rainey, 1948; Leroi-Gourhan, 1935, 1946; Nelson, 1900; Turner, 1889-1890). Entre la parcialidad thule del Ártico central canadiense la caza de ballenas era una práctica obligada durante primavera, época en la que formaban grupos especializados y cooperativos de trabajo para salir en búsqueda de cetáceos, permitiéndoles acumular y almacenar grandes excedentes de comida para los meses más difíciles del año (Bockstone, 1976; Grier, 1999; Jensen, 2012; McCartney, 1980; McCartney y Savelle, 1985; Petitot, 1887).

Los makah de la isla de Vancouver, en la costa noroeste de Norteamérica, establecieron una estrecha relación entre cetáceos y seres humanos. Según los primeros relatos europeos, parte de su economía se volcaba hacia la caza de varias especies de ballenas que cruzaban su territorio, como las grises (Eschrichtius robustus), jorobadas (Megaptera novaeangliae), de aleta (Balaenoptera physalus), franca glaciar (Eubalaena glacialis) y orcas (Orcinus orca), de las cuales aprovechaban la carne y grasa para alimentarse (Cavanagh, 1983 Ms.; Renker, 1997; Waterman, 1920). Para tiempos previos al contacto la situación parece haber sido bastante similar, ya que con seguridad desde al menos los $3500 \mathrm{cal}$ AP sus ancestros cazaron 
cetáceos utilizando arpones de hueso (Huelsbeck, 1988; Losey y Yang, 2007). La intensidad y frecuencia de su caza fue alta. De acuerdo a recuentos estadísticos realizados con documentos históricos, cada comunidad cazaba en promedio cinco a seis especímenes por ańo, mientras que del nivel preeuropeo del sitio de Ozette se logró identificar un número mínimo de 67 individuos consumidos a partir de los restos óseos asociados contextualmente a cabezales de arpón (Huelsbeck, 1988; Renker, 1997). Entre sus vecinos insulares nuu-chah-nulth, las ballenas constituían además íconos representacionales de altísimo valor ideológico en la mantención y sostén de las jerarquías sociales y relaciones de poder dentro de las tribus (Arima, 1988; Coté, 2010; Harkin, 1998; Lantis, 1938; Monks et al., 2001).

En la misma costa oeste, pero algunos cientos de kilómetros al sur, los chumash de Santa Bárbara y los canales de California también cazaron la ballena gris, además de ser especialistas en la captura de delfines, pinnípedos, atunes y peces espada tanto antes como después del contacto europeo (Arnold, 1995; Arnold y Bernard, 2005; Arnold et al., 1997; Bernard, 2004; Colten y Arnold, 1998; Heizer, 1974; Porcasi y Fujita, 2000; Rick y Erlandson, 2002). Dentro de su cosmología, el universo estaba dividido en dos mundos paralelos, el terrestre y el submarino, donde el pez espada ocupaba el lugar privilegiado del humano en el mar como gran cazador; un animal venerado entre los chumash por ser el que hería a las ballenas con su espada, facilitando su caza o llevándolas a varar a la orilla, rondando sobre él mitos y ceremonias, y ocupando un papel especial en las representaciones escultóricas y arte rupestre de este pueblo (Bernard, 2005; Conti y Hudson, 1981; Davenport et al., 1993; Hoover, 1974; Meighan, 2000).

En el otro polo del continente, en las expuestas costas del Cabo de Hornos y en el abrigado canal Beagle al sur de Tierra del Fuego, grupos yámana salían esporádicamente en sus canoas hechas con fragmentos de corteza a cazar ballenas, delfines y lobos marinos gracias a aguzados arpones de hueso, aun cuando lo más común era el aprovechamiento de animales moribundos, enfermos o varados. Seres a los que convirtieron en actores protagónicos en sus principales mitos e imaginario social (Bridges,
1875; Chapman, 2012; Hyades, 1885; Lothrop, 1928; Matial et al., 2007).

\section{Primer ejercicio tipológico: definición del arponaje desde la tecnología comparada}

En todos estos casos históricos y etnográficos la eficiencia de la caza marina dependía de los medios técnicos que disponían para apresar al animal. Al menos desde el precursor trabajo de Otis Tufton Mason (1902) existe cierto consenso acerca de la existencia de tres tipos básicos de dispositivos de penetración para la caza, diferenciados entre sí por su función final (Figura 2) (Bennyhoff, 1950; Lavondès, 1982; Ramseyer, 1988): a) dardos para apuñalar, b) dardos con barbas de retención que además de penetrar quedaban sujetas a la presa, y c) arpones con cabezales desmontables. En el primer caso para herir de muerte a la presa causándole el mayor daño posible, en el segundo, además de herirla que el dispositivo quedara inserto impidiendo que se suelte y aumentar el desangre, mientras que el tercero busca solo capturarla y atraparla sin necesidad de darle muerte.

Técnicamente el arpón sería entonces un instrumento para capturar presas más que para matarlas (Brown, 1967; Julien, 1982; Mason, 1902; Petillon, 2008; Ramseyer, 1988). Un dispositivo más cercano al lazo o al anzuelo que a la lanza y el rifle. Todo sistema de arponaje cuenta para esto con: a) un cabezal desmontable que penetra al animal, b) un astil principal al que se acopla el cabezal, y c) una línea que conecta el cabezal al cazador o a una boya (Figura 3) (Bennyhoff, 1950; Brown, 1967; Leroi-Gourhan, 1935, 1973; Mason, 1902; Skinner, 1937). El método de caza puede variar desde el ataque directamente sobre la presa hasta el uso de un propulsor para arrojar el arpón a la distancia (Gould, 1970; Leroi-Gourhan, 1935, 1973; Mason, 1893, 1902). El arpón se puede definir por tanto, a partir de sus tres funciones básicas: penetrar, quedar sujeto y conectar a la presa con el cazador. Cada una de ellas determina un atributo tecnológico del cabezal desmontable del arpón (Figura 3): g) un extremo penetrante, e) un método de retención en el interior del animal, y f) un sistema de amarre y sujeción entre el cazador y la presa (Stordeur, 1980). 


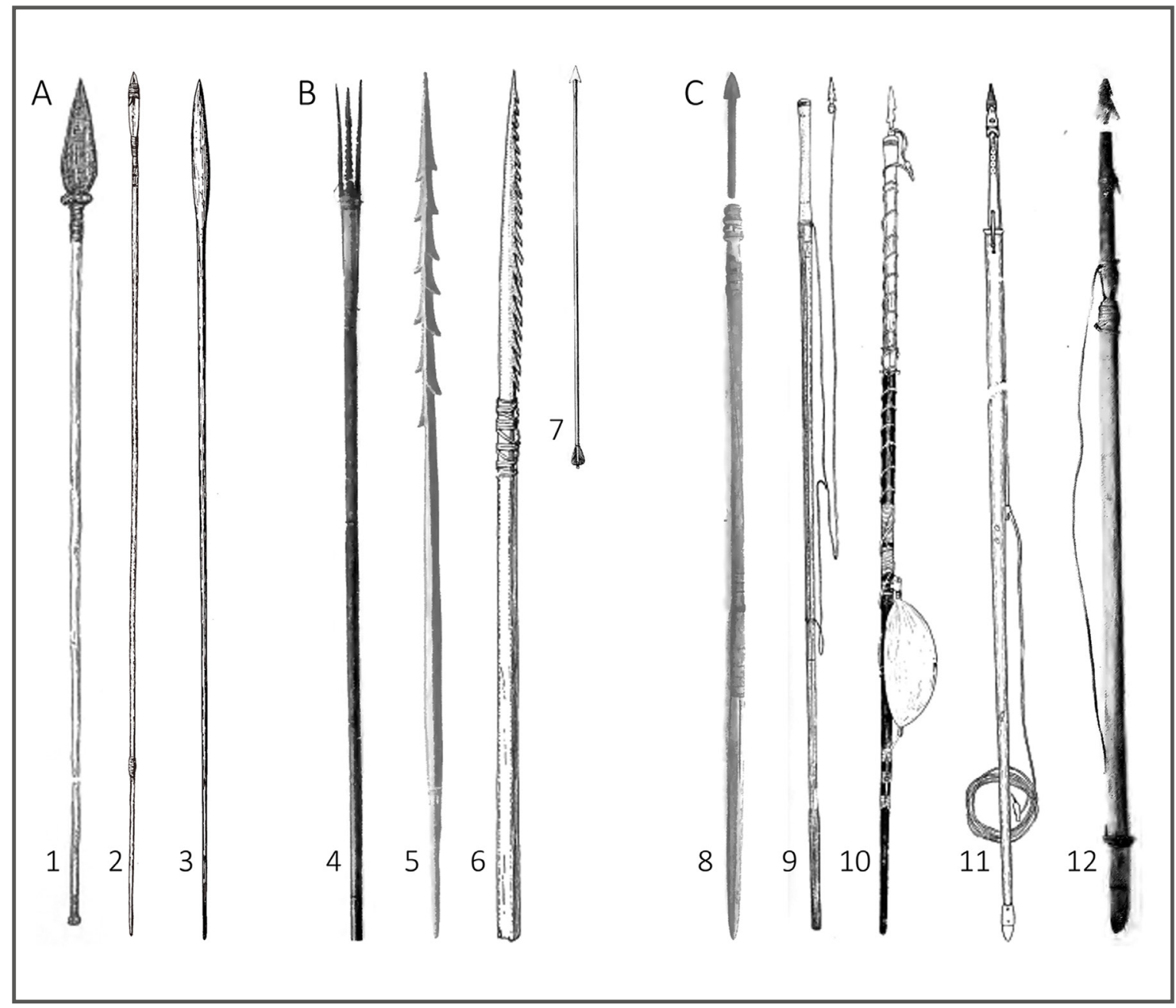

Figura 2. Tipos de dispositivos de penetración para la caza: (A) dardos para apuñalar, (B) dardos con barbas de retención y (C) arpones. Las piezas corresponden a: (1) Venezuela (Mason, 1902, Pl.3); (2) Ngatatjara, desierto australiano (Gould, 1970, Fig.5.a); (3) Ngatatjara, desierto australiano (Gould, 1970, p. 8; Fig.5.b); (4) Negritos, Filipinas (Krieger, 1926a; Pl.3.8); (5); Tule, Panamá (Krieger, 1926b: Pl.11.6); (6) Fueguino, Patagonia (Mason, 1902, Pl.2); (7) Onas, Tierra del Fuego (Lothrop, 1928, Pl.V.a); (8) Eskimo, Nunivak-Alaska (Nelson, 1900, Pl.LV.1); (9) Eskimo, Unalaska-Alaska (Mason, 1902, Pl.18); (10) Eskimo, Bristol Bay-Alaska (Mason, 1902, Pl.13); (11) Eskismo, Groenladia (Mason, 1902, Pl.4); (12) Caleta Vitor, Norte de Chile (Bird, 1946, Pl.123.j). Las piezas no están a escala.

Adicionalmente, todo cabezal posee un mecanismo de acople con el astil principal (Figura 3D). Se conocen dos formas de ensamble, machos y hembras (Lavondès, 1982; Leroi-Gourhan, 1946, 1973). En el primero el cabezal desmontable cuenta con una protuberancia o pedúnculo en su base que se inserta en la cavidad del extremo distal del astil principal, mientras que en el segundo, es el cabezal el que tiene la cavidad y se posa sobre la punta prominente del astil.
Funcionalmente están destinados a la captura de la presa más que a matarla debido a que se utilizan casi exclusivamente en ambientes acuáticos (Julien, 1982; Leroi-Gourhan, 1973; Ramseyer, 1988). Aquí el ser humano al no poder faenar y consumir la presa en el lugar mismo de caza -a diferencia de los animales terrestres- está obligado a trasladarlo hasta su medio, en tierra firme. De ahí que la mayor inversión técnica no se vuelque en la sección de penetración del dispositivo en la presa, sino en su 


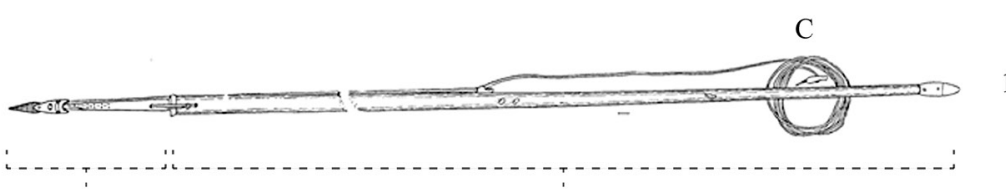

B

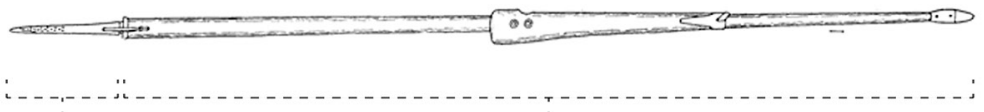

A

B

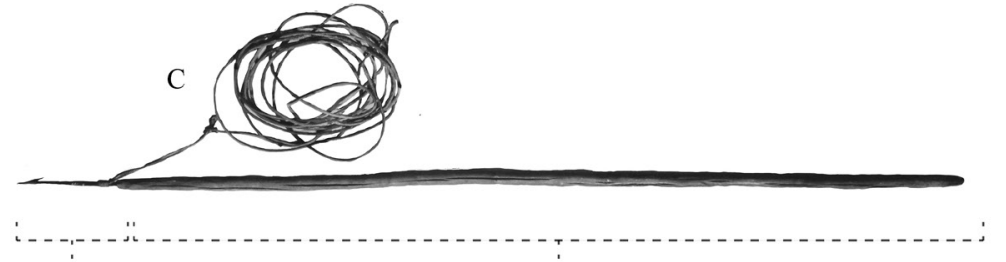

A

B

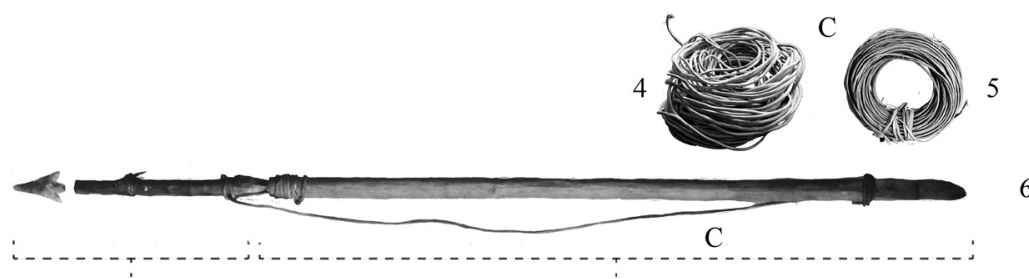

A

B
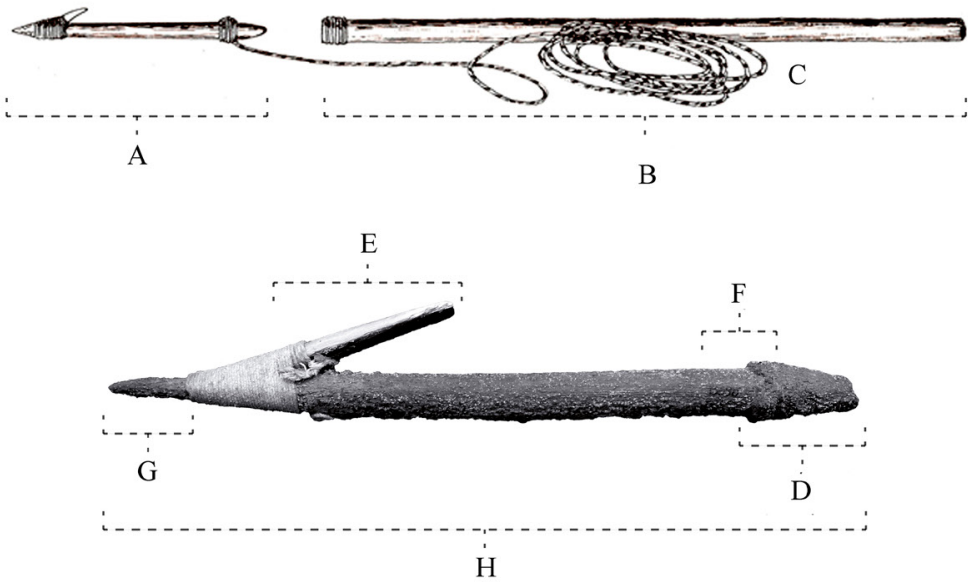

$\mathrm{H}$

Figura 3. Esquema de las partes y secciones que componen el dispositivo de arponaje (1-3, 6-7): (A) Cabezal de arpón, (B) astil principal, y (C) línea de caza. En el cabezal del arpón (8), las secciones son: (D) acople al astil principal, (E) barba de retención a la presa, $(\mathrm{F})$ sistema de retención a la línea de captura, $(\mathrm{G})$ extremo penetrante, $(\mathrm{H})$ vástago del cabezal. Las piezas corresponden a: (1-2) Arpón completo, Eskimo, Groenlandia (Mason, 1902: Pl.4); (3) Arpón completo, Caleta Vitor (Bird, 1946; Pl.123.a); (4) Línea de caza enrollada (50,8 m), Camarones 9 (MASMA) (Ballester, 2017, Fig.3.A); (5) Línea de caza enrollada (71,8 m), Camarones 9 (MASMA) (Ballester, 2017, Fig.3.B); (6) Arpón completo, Caleta Vitor (Bird, 1946,

Pl.123.j); (7) Esquema del arpón para grandes presas (Llagostera, 1989, Fig.2.b); (8) Cabezal de arpón, Punta Blanca,

Tocopilla (MA). Las piezas no están a escala. 
capacidad de no soltarse y mantener el control sobre el animal por parte del cazador, a diferencia quizás de otros dispositivos.

Desde la etnografía y arqueología se han identificado dos tipos generales de cabezales de arpón, en palanca (toggle) y simples-barbados (Arnold, 1989; Bennyhoff, 1950; Leroi-Gourhan, 1946, 1973; Mason, 1902; Stordeur, 1980). Los cabezales en palanca son dispositivos que al penetrar en la presa, giran y quedan perpendiculares a la línea de sujeción, impidiendo que éste se suelte. Poseen por lo general una sección penetrante y un orificio medial que asegura el amarre de la línea a la vez que permitir el giro del cabezal y funcionar como palanca en forma de T (Figura 4B) (Arnold, 1989; Brown, 1967; Collins, 1941; Larsen y Rainey, 1948; Lavondès, 1982; Megginson, 2000; Park y Mousseau, 2003; Quimby, 1946; Skinner, 1937; Wissler, 1916; Yama’ura, 1984).

Los cabezales simples-barbados se diferencian en que su método de retención a la presa depende de una o varias barbas laterales que al insertarse quedan en una posición paralela o diagonal al eje de la línea de sujeción, funcionando de gancho para quedar aferrado al interior del animal (Figura 4A) (Bennyhoff, 1950; Beauchamp, 1902; Black, 1890; Lavondès, 1982; Märgärit et al., 2010; Ramseyer, 1988; Read, 1890; Weniger, 1992, 2000; Wissler, 1916; Wintemberg, 1906; Yellen, 1998). Dentro de esta categoría existen varias modalidades, como los cabezales barbados de una sola pieza que son manufacturados desde un mismo soporte o base ( $\mathrm{Fi}$ gura $4 \mathrm{C}$ ), y los cabezales compuestos que reúnen varias secciones independientes unidas entre sí para dar forma al cabezal final (Figura 4D) (Bennyhoff, 1950).

$\mathrm{Al}$ interior de cada una de estas clases generales pueden establecerse distinciones según sus rasgos morfológicos particulares, como el número y lateralidad de las barbas, materia prima y tamaño de las distintas piezas que las componen, las características de la sección penetrante y forma de amarre a la línea de sujeción, por nombrar algunos ejemplos (LeroiGourhan, 1946; Stordeur, 1980; Weniger, 1992). Cada uno de estos elementos dependerá de la dis- ponibilidad de materias primas, morfología de los soportes, estrategias de caza, tipo de presa, junto a decisiones estilísticas y de diseño de carácter cultural independientes de cada grupo.

\section{Arpones del desierto de Atacama: universo material y muestra de análisis}

Aunque la evidencia arqueológica de arpones es fragmentaria e incompleta en el litoral del desierto de Atacama -como casi siempre en arqueología-, el dispositivo utilizado en momentos prehispánicos parece haber sido semejante al descrito para tiempos coloniales (Bird, 1943, 1946; Digby, 1934). Mientras en los grandes conchales litorales solo se han recuperado secciones parciales y fracturadas, especialmente barbas de hueso, cabezales líticos y trozos de vástagos, ha sido en los contextos fúnebres donde se han recobrado piezas integrales, con sus partes asociadas y en contextos definidos aunque, claro está, con el sesgo que ha producido el intenso saqueo de los cementerios.

Considerando estas limitaciones de registro, en el norte de Chile el dispositivo de arponaje se compone en general de tres secciones (Figura 3): a) un cabezal desmontable que penetra en la presa, b) un astil principal cargado por el cazador en donde va acoplado el cabezal, y c) una línea de caza que une el cabezal con el cazador (Bird, 1943, 1946; Llagostera, 1989; Núñez, 1999). Los dos primeros complementos son a su vez compuestos. El cabezal cuenta con un vástago central, una o dos barbas laterales ubicadas hacia la sección distal, en ciertas ocasiones una punta lítica en el extremo penetrante y en algunos casos encordados perimetrales de fibras vegetales. El astil principal puede alcanzar los $3 \mathrm{~m}$ de longitud gracias a un sistema de ensamblaje de hasta tres partes, unidas entre sí de dos formas, sea mediante el sistema desmontable macho-hembra de pedúnculo-cavidad tal como en los cabezales, o con cortes biselados en zigzag que calzan a la perfección para luego ser firmemente encordados (Ballester y Clarot, 2014; Bird, 1943, 1946; Ewbank, 1855; Figueroa et al., 2015; Focacci, 1974; Focacci y Chacón, 1989; Latcham, 1910; Llagostera, 1989; Mostny, 1964; Núñez, 1999; Spahni, 1967; Uhle, 1917, 


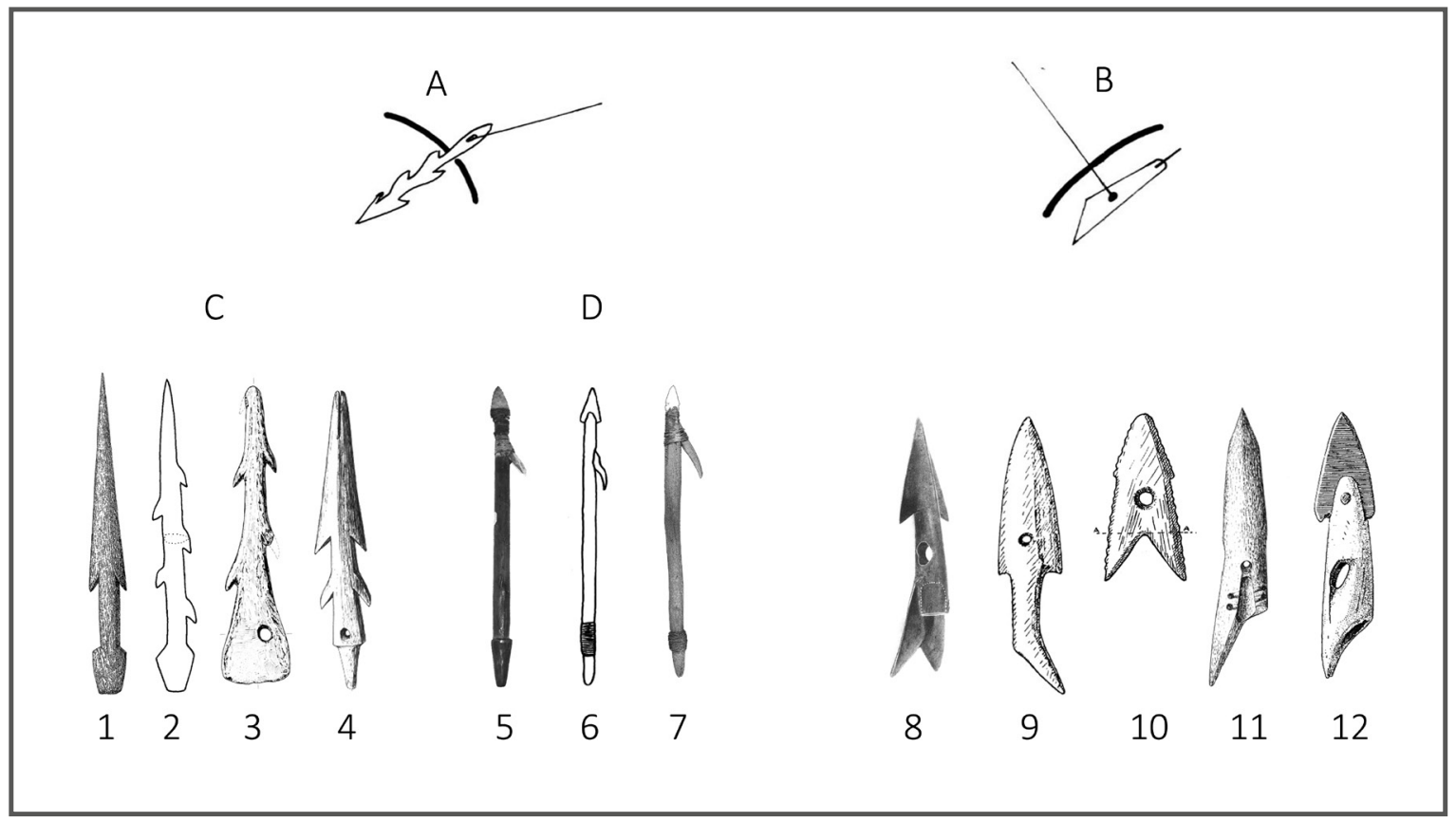

Figura 4. Tipología de cabezales de arpón: (A) simple-barbado y (B) en palanca (Lavondès, 1982, Fig.2); (C) barbados de una sola pieza y (D) barbados compuestos. Las piezas corresponden a: (1) Yámana, Tierra del Fuego (Hyades, 1885, Fig. 185); (2) costa de California (Bennyhoff, 1950, Fig.4.a); (3) Horgen, Suiza (Ramseyer, 1988, Fig.4.a); (4) Eskismo, Point Hope-Alaska (Wissler, 1916, Fig.15. b); (5) Chumash, California (Read 1892: Pl.XL.2); (6) Caleta Huelén, Norte de Chile (Spahni, 1967, Pl.III.14); (7) La Chimba, Norte de Chile (MQB); (8) Nueva Escocia, Norteamérica (Gudger, 1942, p. 420); (9) Maorí, Nueva Zelanda (Skinner, 1937, Fig.1); (10) Maorí, Polinesia francesa (Skinner, 1937, Fig.18); (11) Eskismo, Langton BayAlaska (Wissler, 1916, Fig.22); (12) Eskismo, Groenlandia (Mason, 1902, Fig.24). Las piezas no están a escala.

1922). La única sección del equipo no compuesta era la línea de caza, manufacturada como una sola pieza sin amarras intermedias a partir de un cuero de lobo marino cortado delgadamente con finas terminaciones en sus extremos, pudiendo a llegar a medir $70 \mathrm{~m}$ de largo contenidos en gruesos rollos (Ballester, 2017; Spahni, 1967).

A diferencia de lo que ocurre en los Valles Occidentales del extremo norte, el litoral antofagastino cuenta con un modesto registro de arpones debido a la falencia de investigaciones dirigidas y producto del abandono y enorme saqueo. Nuestros esfuerzos iniciales fueron dirigidos a la búsqueda, registro, contextualización y análisis de las piezas de arpón depositadas en los museos de la región, sin olvidar aquellas trasladadas hacia otras latitudes tanto nacionales como extranjeras, junto a la información disponible en publicaciones científicas y documentos de excavación.
En total logramos registrar más de 300 cabezales de arpón, de los cuales solo 129 contaban con información de procedencia y contexto que permitiera atribuirlos a un sitio y período (Tabla 1 ). Su contextualización en ciertos casos requirió reestudiar la totalidad de las colecciones del sitio, conseguir los cuadernos y notas de campo, entrevistar a quienes participaron de las excavaciones y realizar nuevos fechados radiocarbónicos de las piezas. De este modo, datamos como parte de esta investigación 59 nuevos cabezales de arpón, lo que sumado a trabajos anteriores forma un conjunto compuesto de 31 cabezales provenientes de un contexto fúnebre datado, 43 de un cementerio datado y 55 con adscripción temporal según los rasgos culturales del contexto. Solamente en 40 de los ejemplares se pudo obtener información bioantropológica de los individuos a los cuales estaban ofrendados, en todos los casos hombres adultos. La colección proviene de contextos fúnebres de toda la prehistoria del litoral, 
ubicados geográficamente desde el norte de la desembocadura del río Loa $\left(21^{\circ} 21^{\prime} S\right)$ hasta el sur de la localidad de Taltal $\left(25^{\circ} 24^{\prime} \mathrm{S}\right)$, comprendiendo más de $450 \mathrm{~km}$ en línea recta de costa (Figura 5, Tabla 1). Hasta el momento no fue registrada ninguna pieza completa procedente del interior del desierto dentro de las latitudes estudiadas, volviéndolos un bien esencialmente costero. ${ }^{2}$

Los cabezales se analizaron directamente en colecciones de museos e indirectamente desde información en publicaciones científicas cuando éstas lo permitían, registrando los atributos morfológicos, técnicos y estilísticos mencionados en la sección anterior, junto a las medidas de largo, diámetro menor y mayor de cada pieza. Se consideraron únicamente instrumentos que contaran con los elementos técnicos atribuibles a la categoría de cabezales de arpón, como son la barba de retención, sistema de amarre a la línea de captura y/o acople para un astil principal (Figura 3E, F y D), con tal de no generar confusiones con otros tipos de artefactos de similar forma, pero distinta función (p.e., barbas de poteras, agujas, punzones) (Barraza, 1981; Labarca et al., 2017; Silva y Bahamondes, 1968).

\section{Segundo ejercicio tipológico: arpones de la costa del desierto de Atacama}

La totalidad de los cabezales estudiados corresponden al tipo "simple-barbado compuesto" con un sistema de acople "macho" (Figura 4D), formado siempre por al menos un vástago hecho de una sola pieza, una o dos barbas independientes adosadas a un costado gracias a una atadura de cordelería, incluyendo en algunos casos, además, una punta lítica en la sección distal penetrante y un sistema de amarre perimetral en la sección proximal para

2 Si bien Núñez $(1962,2006)$ recuperó cabezales de arpón en Pica, Tarapacá y salar de Soronal, en la región de Tarapacá, ésta quedó fuera de los límites geográficos de este análisis debido a que representa una realidad social diferente de la cuenca del Loa hacia el sur, con otras lógicas de movilidad y relaciones comunitarias. Una situación similar ocurre con los cabezales recuperados por Latcham (1938) en Quillagua, piezas que hasta el momento no tienen contexto de proveniencia conocido. contener la línea de retención de la presa. Esta situación marca una primera norma tecnológica de cierto nivel de estandarización en los diseños y su manufactura.

Pero aquello que desde una primera mirada general parece homogéneo y similar, al revisar en detalle sus principales rasgos tecnológicos surgen diferencias y variantes que requieren de un ordenamiento y clasificación. Para este segundo ejercicio tipológico hemos definido cuatro atributos principales del arpón que nos parecen son los más significativos en términos tecnológicos relativos a su funcionamiento y uso, cada uno con distintas variantes técnicas.

El primer atributo es el extremo penetrante, sección encargada de hacer el primer encuentro con el animal cazado y de cuya morfología depende la eficacia de penetración en los tejidos de la presa. Para este atributo técnico registramos dos variantes de manufactura, aquellas piezas cuyo vástago fue modelado en su extremo distal por façonnage para dejar una ranura transversal al eje donde se insertaba una punta lítica, en los casos de mejor conservación adherida con una goma (Figura 6E2). En ciertas ocasiones uno de los costados de la ranura se extiende más hacia el proximal, dejando una cavidad prolongada sobre la cara donde se inserta la barba lateral de hueso. La otra variante se caracteriza por piezas cuyo vástago fue tallado y pulido para dejar el extremo penetrante de forma puntiaguda y aguzada, de sección semicircular o con caras biseladas y aplanadas (Figura 6E1).

El segundo atributo corresponde al sistema de retención del cabezal al interior del animal, en este caso la barba de retención, rasgo técnico de vital importancia considerando que la función primordial del arpón es lograr capturar la presa sin que se libere. Dentro del conjunto solo se registraron dos soluciones técnicas, aunque siguiendo un diseño bastante similar. El caso más común es la variante que presenta una sola barba lateral adosada al vástago a través de un hilado perimetral con fibras (Figura 6B2), piezas manufacturadas principalmente de hueso de camélido, trabajadas finamente por façonnage mediante raspado y pulido, con un bisel alargado en el extremo para el acople con el vástago, un cuerpo curvo y de sección subcircular que deja un ángulo 
Tabla 1. Muestra de cabezales de arpón con información contextual y atributos técnicos.

\begin{tabular}{|c|c|c|c|c|c|c|c|c|c|c|c|c|c|c|c|c|c|c|}
\hline \multicolumn{2}{|l|}{ Contexto } & \multirow{2}{*}{$\begin{array}{c}\text { Cronología } \\
\text { Relativa }\end{array}$} & \multirow{2}{*}{$\begin{array}{l}\text { Núm. } \\
\text { total }\end{array}$} & \multicolumn{3}{|c|}{ Extremo penetrante } & \multicolumn{3}{|c|}{ Barba retención } & \multicolumn{3}{|c|}{ MP vástago } & \multicolumn{4}{|c|}{ Retención de la línea } & \multirow{2}{*}{$\begin{array}{c}\text { Tipo } \\
\text { arpón }\end{array}$} & \multirow[b]{2}{*}{ Referencia } \\
\hline Sitio & Unidad & & & E1 & E2 & Ind. & B1 & B2 & Ind. & $\mathrm{Al}$ & A2 & Ind. & RL1 & RL2 & RL3 & Ind. & & \\
\hline Agua Dulce & - & PIT & 2 & 2 & - & - & - & - & 2 & 2 & - & - & 2 & - & - & - & A & Mostny, 1964 \\
\hline La Chimba & - & PIT & 2 & 2 & - & - & - & - & 2 & 2 & - & - & 2 & - & - & - & A & $\mathrm{MQB}$ \\
\hline Chipana & - & PIT & 2 & 2 & - & - & 2 & - & - & 2 & - & - & 2 & - & - & - & A & Digby, 1934 \\
\hline Chipana & - & Colonial & 1 & 1 & - & - & 1 & - & - & 1 & - & - & 1 & - & - & - & A & Digby, 1934 \\
\hline Las Loberas & T. 1 & Formativo & 1 & 1 & - & - & 1 & - & - & 1 & - & - & 1 & - & - & - & A & Ballester \& Clarot, 2014 \\
\hline $\mathrm{CaH} 42$ & - & Arcaico Tardío & 1 & 1 & - & - & 1 & - & - & 1 & - & - & 1 & - & - & - & A & $\mathrm{UA}$ \\
\hline $\mathrm{CaH} 12$ & - & PIT & 1 & 1 & - & - & 1 & - & - & 1 & - & - & 1 & - & - & - & A & UA \\
\hline $\mathrm{CaH} 02$ & T. 3 & PIT & 1 & 1 & - & - & 1 & - & - & 1 & - & - & 1 & - & - & - & A & Spahni, 1967 \\
\hline $\mathrm{CaH} 31$ & T. 22 & Formativo & 1 & 1 & - & - & 1 & - & - & 1 & - & - & 1 & - & - & - & A & Spahni, 1967 \\
\hline $\mathrm{CaH} 10$ & - & Formativo & 1 & - & - & 1 & - & - & 1 & - & 1 & - & - & 1 & - & - & B & Fondecyt 1110702 \\
\hline Punta Guaque 02 & T. 1 & Formativo & 1 & - & 1 & - & - & - & 1 & - & 1 & - & - & 1 & - & - & B & Fondecyt 1110702 \\
\hline Punta Tamira 02 & - & Formativo & 1 & - & 1 & - & - & - & 1 & - & 1 & - & - & 1 & - & - & $\mathrm{B}$ & Fondecyt 1110702 \\
\hline Punta Blanca 01 & T. 2 & Formativo & 3 & 1 & 2 & - & - & 2 & 1 & - & 3 & - & - & 3 & - & - & B & MA \\
\hline $\mathrm{CaH} 20$ & - & Formativo & 3 & 2 & 1 & - & - & - & 3 & - & 3 & - & - & 3 & - & - & B & UA \\
\hline $\mathrm{CaH} 20$ & T. 30 & Formativo & 1 & - & 1 & - & - & - & 1 & - & 1 & - & - & 1 & - & - & B & Spahni, 1967 \\
\hline $\mathrm{CaH} 02$ & T. 16 & PIT & 1 & - & 1 & - & - & - & 1 & - & 1 & - & - & 1 & - & - & B & Spahni, 1967 \\
\hline $\mathrm{CaH} 02$ & T. 20 & PIT & 2 & 2 & - & - & - & 1 & 1 & - & 2 & - & - & 2 & - & - & B & Spahni, 1967 \\
\hline $\mathrm{CaH} 04$ & T. 10 & PIT & 3 & - & 3 & - & - & 2 & 1 & - & 3 & - & - & 2 & - & 1 & B & Spahni, 1967 \\
\hline $\mathrm{CaH} 02$ & - & PIT & 1 & - & - & 1 & - & - & 1 & - & 1 & - & - & 1 & - & - & B & Fondecyt 1110702 \\
\hline $\mathrm{CaH} 07$ & A & Formativo & 4 & - & 3 & 1 & - & - & 4 & - & 4 & - & - & 4 & - & - & B & Fondecyt 1110702 \\
\hline $\mathrm{CaH} 07$ & B & Formativo & 3 & - & 1 & 2 & - & - & 3 & - & 3 & - & - & 3 & - & - & B & Fondecyt 1110702 \\
\hline $\mathrm{CaH} 07$ & $\mathrm{D}$ & Formativo & 1 & - & - & 1 & - & - & 1 & - & 1 & - & - & 1 & - & - & B & Fondecyt 1110702 \\
\hline $\mathrm{CaH} 07$ & E & Formativo & 1 & - & - & 1 & - & - & 1 & - & 1 & - & - & 1 & - & - & B & Fondecyt 1110702 \\
\hline $\mathrm{CaH} 07$ & $\mathrm{~F}$ & Formativo & 1 & - & 1 & - & - & - & 1 & - & 1 & - & - & 1 & - & - & B & Fondecyt 1110702 \\
\hline $\mathrm{CaH} 10 \mathrm{~A}$ & $\mathrm{C}$ & Formativo & 2 & - & 1 & 1 & - & - & 2 & - & 2 & - & - & 2 & - & - & B & Fondecyt 1110702 \\
\hline $\mathrm{CaH} 20$ & $\mathrm{~J}$ & Formativo & 1 & - & - & 1 & - & - & 1 & - & 1 & - & - & 1 & - & - & B & Fondecyt 1110702 \\
\hline $\mathrm{CaH} 51$ & - & PIT & 9 & 3 & 1 & 5 & - & - & 9 & - & 9 & - & - & 9 & - & - & B & Fondecyt 1110702 \\
\hline $\mathrm{CaH} 53$ & - & Colonial & 4 & 2 & 1 & 1 & - & - & 4 & - & 4 & - & - & 4 & - & - & B & Fondecyt 1110702 \\
\hline Las Loberas & T. 1 & Formativo & 3 & - & 3 & - & - & - & 3 & - & 3 & - & - & 3 & - & & B & Ballester \& Clarot, 2014 \\
\hline $\mathrm{CaH} 04$ & T. 5 & PIT & 1 & - & 1 & - & - & 1 & - & - & 1 & - & - & 1 & - & - & B & Spahni, 1967 \\
\hline Caleta Oliva & - & Formativo & 3 & - & 3 & - & - & 3 & - & 3 & - & - & - & - & 3 & - & $\mathrm{C}$ & Capdeville, 1928; Mostny, 1964 \\
\hline Cementerio ollita gris & - & Formativo & 1 & - & 1 & - & - & 1 & - & 1 & - & - & - & - & 1 & - & $\mathrm{C}$ & Mostny, 1964 \\
\hline Las Guaneras & - & Formativo & 2 & - & 2 & - & - & 2 & - & 2 & - & - & - & - & 2 & - & $\mathrm{C}$ & Mostny, 1964 \\
\hline Puntilla Sur Vaso figurado & - & Formativo & 3 & - & 3 & - & - & 3 & - & 3 & - & - & - & - & 3 & - & $\mathrm{C}$ & Mostny, 1964 \\
\hline Gualaguala 04 & F05 & Formativo & 1 & - & - & 1 & - & - & 1 & 1 & - & - & - & - & 1 & - & C & Fondecyt 1110702 \\
\hline Michilla 02 & T. 5 & Formativo & 1 & - & - & 1 & - & - & 1 & 1 & - & - & - & - & 1 & - & $\mathrm{C}$ & Fondecyt 1110702 \\
\hline Michilla 02 & T.7 & Formativo & 1 & - & 1 & - & - & 1 & - & 1 & - & - & - & - & 1 & - & $\mathrm{C}$ & Fondecyt 1110702 \\
\hline El Vertedero Antofagasta & T. $1-2$ & Formativo & 3 & 2 & - & 1 & - & 2 & 1 & 3 & - & - & - & - & 2 & 1 & $\mathrm{C}$ & MA \\
\hline Punta Blanca 2 & T. 4 & Formativo & 1 & 1 & - & - & - & - & 1 & 1 & - & - & - & - & 1 & - & $\mathrm{C}$ & MA \\
\hline Punta Guaque 02 & T. 6 & Formativo & 1 & - & - & 1 & - & - & 1 & 1 & - & - & - & - & 1 & - & $\mathrm{C}$ & Fondecyt 1110702 \\
\hline Las Loberas & T. 1 & Formativo & 4 & 4 & - & - & - & 4 & - & 4 & - & - & - & - & 4 & - & $\mathrm{C}$ & Ballester \& Clarot, 2014 \\
\hline La Chimba & - & PIT & 7 & - & 4 & 3 & - & 3 & 4 & - & 7 & - & 7 & - & - & - & $\mathrm{D}$ & Quai Branly \\
\hline Auto Club & T. 4 & PIT & 2 & - & 2 & - & - & - & 2 & - & 2 & - & 2 & - & - & - & $\mathrm{D}$ & Ballester et al., 2014a \\
\hline Auto Club & T. 11 & PIT & 13 & 1 & 11 & 1 & - & 1 & 12 & - & 13 & - & 5 & - & - & 8 & $\mathrm{D}$ & Ballester et al., 2014a \\
\hline Auto Club & T. 12 & PIT & 4 & - & 3 & 1 & - & 3 & 1 & - & 4 & - & - & - & - & 4 & $\mathrm{D}$ & Ballester et al., 2014a \\
\hline $\mathrm{CaH} 33$ & T. 7 & PIT & 3 & - & 3 & - & - & 1 & 2 & - & 3 & - & 1 & - & - & 2 & $\mathrm{D}$ & Spahni, 1967 \\
\hline $\mathrm{CaH} 04$ & T. 5 & PIT & 2 & - & 2 & - & - & 1 & 1 & - & 2 & - & 2 & - & - & - & $\mathrm{D}$ & Spahni, 1967 \\
\hline $\mathrm{CaH} 04$ & T. 9 & PIT & 1 & - & 1 & - & - & - & 1 & - & 1 & - & 1 & - & - & - & $\mathrm{D}$ & Spahni, 1967 \\
\hline $\mathrm{CaH} 04$ & T. 5 & PIT & 8 & - & 8 & - & - & 1 & 7 & - & 8 & - & - & - & - & 8 & - & Spahni, 1967 \\
\hline $\mathrm{CaH} 04$ & T. 15 & PIT & 3 & - & 3 & - & - & 3 & - & - & 3 & - & - & - & - & 3 & - & Spahni, 1967 \\
\hline $\mathrm{CaH} 04$ & T. 17 & PIT & 1 & - & 1 & - & - & 1 & - & - & 1 & - & - & - & - & 1 & - & Spahni, 1967 \\
\hline $\mathrm{CaH} 07$ & $\mathrm{C}$ & Formativo & 1 & - & - & 1 & - & - & 1 & - & 1 & - & - & - & - & 1 & - & Fondecyt 1110702 \\
\hline Choluto & - & Formativo & 2 & - & 2 & - & - & 2 & - & - & 2 & - & - & - & - & 2 & - & Castelleti, 2007 \\
\hline Cobija 10 & - & Formativo & 2 & 1 & 1 & - & - & 1 & 1 & 1 & 1 & - & - & - & - & 2 & - & Moragas, 1982 \\
\hline
\end{tabular}




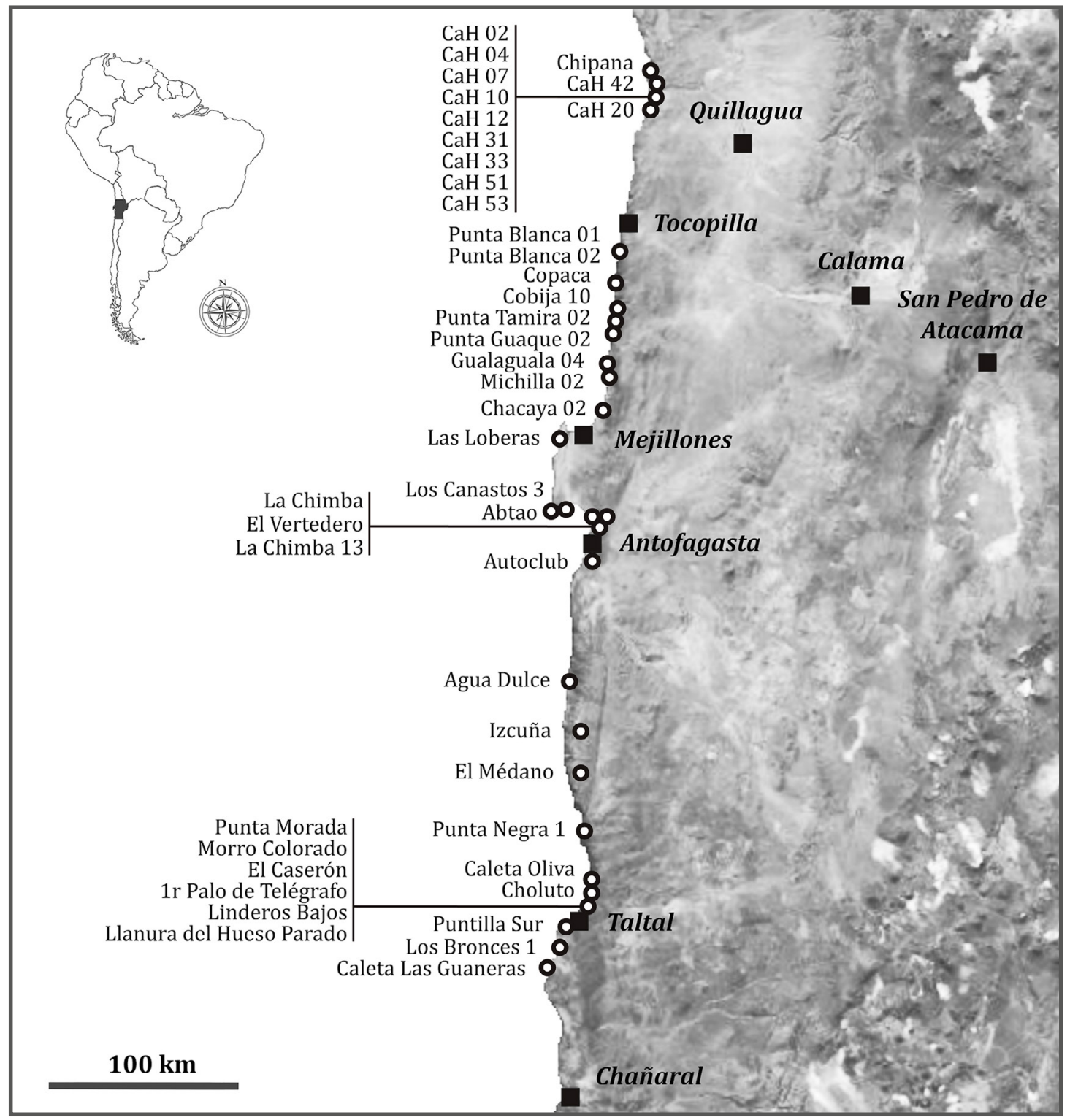

Figura 5. Ubicación geográfica de sitios arqueológicos mencionados en el texto.

variable entre $45^{\circ}-20^{\circ}$ respecto del eje del cabezal. El tamaño, la forma y sección de estas barbas puede variar, pero el esquema general de ser una sola, lateral y de hueso de camélido se mantiene. La segunda variante presenta siempre dos barbas alargadas paralelas una al lado de la otra, amarradas también mediante fibras y dispuestas cercanas al extremo distal de la pieza (Figura 6B1). Las barbas son rectas y alargadas, ambas del mismo tamaño, formando un ángulo respecto del eje del cabezal notoriamente menor al caso anterior, por lo general utilizando es- pinas de cactus para su manufactura, aunque existen unos pocos casos con lancetas de cobre que imitan su forma.

El tercer atributo corresponde a la materia prima escogida para la fabricación del vástago del cabezal, elemento técnico que será fundamental en la resistencia mecánica, flexibilidad y peso del dispositivo. Una parte del conjunto utilizó materias primas óseas, implicando etapas previas de selección de nódulos para su posterior debitage y façonnage (Figura 


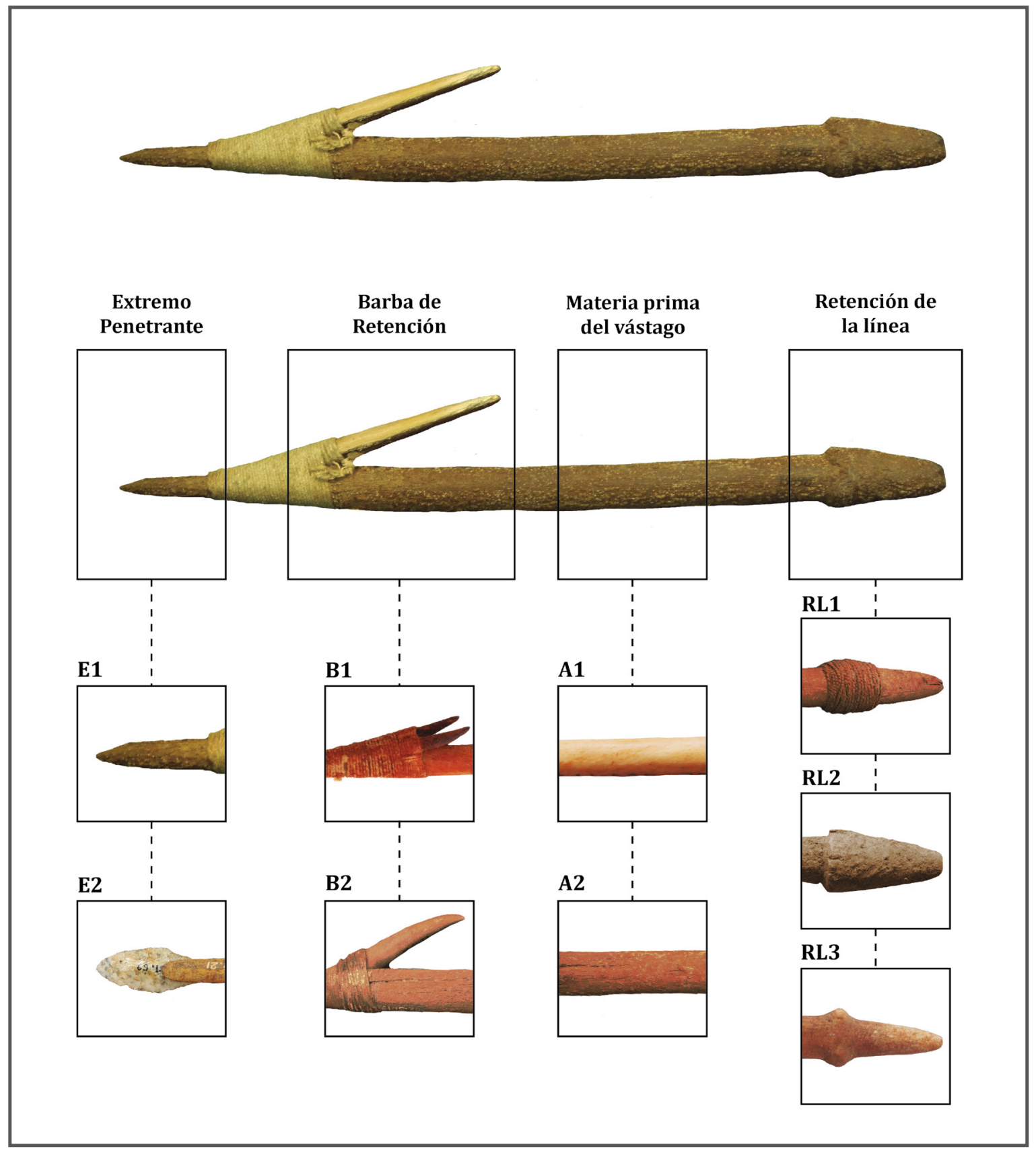

Figura 6. Principales atributos tecnológicos y sus variantes en los cabezales de arpón del desierto de Atacama. Extremo penetrante: (E1) aguzado y (E2) cabezal lítico. Barba de retención: (B1) barbas paralelas de espinas de cactus o cobre y (B2) barba única de hueso. Materia prima del vástago: (A1) hueso y (A2) madera. Retención de la línea de caza: (RL1) encordelado perimetral, (RL2) modelado cónico y (RL3) engrosamiento. 
6A1). Otro grupo fue manufacturado en madera, un recurso que si bien es escaso en el desierto fue sumamente importante para las poblaciones litorales (Figura 6A2). Para cada variante, óseo y madera, se utilizaron distintos recursos animales y forestales para los soportes, lo que como veremos más adelante tuvo importantes implicancias tecnológicas.

El último atributo es el modo de retención de la línea de caza, solución técnica empleada para que el amarre de la línea no se soltara del cabezal asegurando la retención de la presa, así como la recuperación del cabezal en actos fallidos. En el conjunto se registraron tres variantes. La primera ocupa una solución técnica aditiva o compuesta, ya que en la sección proximal del vástago del cabezal se adhiere un encordado perimetral de fibras de varias vueltas que genera una especie de anillo firme y rígido que servirá de tope para la amarra de la línea de caza, en general de algodón hilado y trenzado (Figura 6RL1). En las otras dos variantes la retención de la línea se realizó modelando por façonnage la sección proximal del vástago hasta formar un engrosamiento de mayor diámetro que impedía la soltura de la amarra de la línea. Así, en la segunda variante el esculpido fue hecho según un diseño cónico de extremo truncado que servía en su sección más gruesa para frenar la línea y en la más delgada o aguzada para calzar por presión y quedar sujeta en la cavidad hembra del astil principal del arpón (Figura 6RL2). La tercera y última variante presenta un modelado para formar un engrosamiento en su sección proximal del vástago que retiene la línea de caza (Figura 6RL3), en general en forma de anillo rodeando todo su perímetro o como protuberancias laterales (cruceta).

No todos los atributos son igual de importantes en la tecnología de arponaje. Como revisamos, los más significativos son la barba de retención y el modo de retención de la línea de caza debido a su papel protagónico en la función principal del mecanismo para quedar inserto en la presa y evitar que la línea se suelte. Así, los atributos y su combinación se jerarquizaron en la construcción de la tipología (Read 2007), considerando en el nivel más alto la barba de retención, luego el sistema de retención de la línea de caza, seguido de la materia prima del vástago y finalmente la forma del extremo penetrante. La combinación de estas variantes técnicas entre sí fue limitada y recurrente. De 24 posibles combinaciones entre las variantes de los cuatro atributos considerados $(2 \times 3 \times 2 \times 2)$, solo se formaron cuatro clases (Figura 7), una de ellas con dos subtipos, en total cinco combinaciones posibles correspondiente al $20,8 \%$ de las probabilidades.

El Tipo A (Figura 8A) es un cabezal compuesto de un vástago hecho sobre un fragmento longitudinal de metapodio de camélido, en muchos casos exhibiendo aún lateralmente la acanaladura medular interior del hueso. El extremo distal es aguzado por raspado y luego pulido en el proceso de façonnage, de sección subcircular a lo largo de toda la pieza y redondeado a través de las mismas técnicas en su extremo proximal. Hacia distal y próximo al extremo penetrante cuenta siempre con dos barbas paralelas, una al lado de la otra en ángulo agudo respecto del eje del cabezal, amarradas entre sí al vástago gracias a un encordado perimetral ancho de fibras de algodón para asegurar la sujeción. Las barbas en la totalidad de los casos estudiados fueron manufacturadas con espinas de cactus previamente seleccionadas, cortadas a la medida y pulidas para dejar el extremo aguzado aunque grueso, a diferencia de su forma original cuyo extremo es delgado y frágil. Tanto en la literatura como en el conjunto que no utilizamos como muestra debido a falta de contexto existen ejemplares en los que se utilizaron barbas aguzadas y pulidas de cobre asimilando la forma de las espinas (Digby, 1934; Figueroa et al., 2015; Mostny, 1964; Salazar et al., 2010).

El Tipo B (Figura 8B) presenta un vástago hecho siempre de madera densa de sección subcircular relativamente homogénea, tallado y raspado. Su rasgo técnico distintivo se ubica en la sección proximal del vástago, donde por façonnage se modeló el soporte para dejar un cono de extremo truncado que sirve para impedir el desliz de la línea de caza y a la vez para insertarse en la cavidad hembra del astil principal. En todos los casos el tipo de retención al interior del animal fue una única barba lateral hecha sobre hueso de camélido ubicado hacia distal del vástago, ocupando el último tercio de la pieza, y adosada con un encordado perimetral de fibras vegetales, principalmente algodón. En algunos casos el vástago presentaba un pequeńo bisel o cavidad para acoplar la barba. Dos subtipos pudieron distinguirse en esta clase, una variante con el extremo distal agu- 
zado y terminado en punta por façonnage (Figura $8 \mathrm{~B} 1$ ), y otra que cuenta con una ranura transversal y a lo ancho del vástago donde se insertaba una punta lítica adherida con una goma y en ciertas ocasiones también con un encordado perimetral de fibras de algodón (Figura 8B2).

El Tipo C cuenta con un vástago hecho sobre hueso de mamífero marino modelado en façonnage mediante tallado, raspado y pulido, dándole una forma final semicilíndrica alargada, siempre con una ranura transversal en su extremo distal para la inserción de una punta lítica (Figura 8C). En su extremo opuesto, para la retención de la línea de caza se labró un engrosamiento del vástago en forma de anillo perimetral o como dos notorias protuberancias laterales (cruceta), en ambos casos rasgos manufacturados en el mismo momento de modelamiento del vástago. El sistema de retención al interior del animal siempre es mediante una única barba lateral de hueso de camélido adosada a través de un encordado perimetral de fibras.
Finalmente el Tipo D se compone de un vástago hecho sobre madera densa, modelado por façonnage hasta lograr una forma semicilíndrica de sección subcircular a lo largo de su eje, dejando siempre una ranura transversal en su extremo distal para la inserción de una punta lítica sujeta a través de una goma (adhesivo) y en ciertas ocasiones además con un encordado de fibras vegetales. En algunos ejemplares la ranura se extiende y prolonga en uno de los lados, dejando una cavidad alargada donde calzar la única barba lateral, ésta siempre sobre hueso de camélido. En su extremo opuesto para la retención de la línea de caza, cuenta con un firme y grueso encordado perimetral con fibras de algodón, y el vástago termina redondeado para calzar en la cavidad hembra del astil principal.

En términos métricos, los cuatro tipos de cabezales presentan diferencias (Figura 9). Los del Tipo A son notoriamente menores y más homogéneos en tamaño que las otras tres variantes, con rangos máximos por debajo de los mínimos de las piezas de los tipos
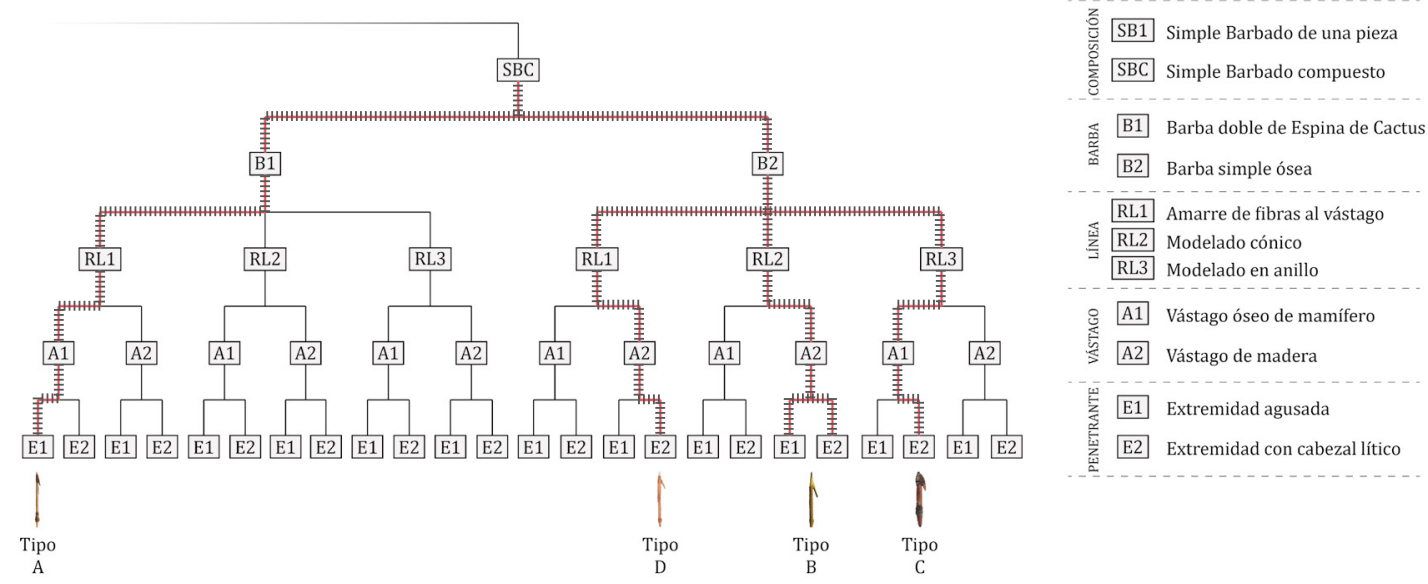

Figura 7. Clasificación tipológica por atributos técnicos jerarquizados. 
B y D. Solamente con el Tipo C hay cierta concurrencia de tamaños. Los tipos $\mathrm{B}$ y $\mathrm{D}$ poseen prácticamente los mismos rangos máximos y mínimos de longitud, con mínimas muy similares cercanas a los $17 \mathrm{~mm}$ justo por encima del máximo del Tipo A $(16,7 \mathrm{~mm})$. El Tipo C, por su parte, es un cabezal de dimensiones intermedias entre el A y los B-D. Estas diferencias métricas tan marcadas entre los tipos $\mathrm{A}$, C y B-D vuelven más significativas las clasificaciones iniciales, distinciones de tamaño que bien pudieron tener relación con el tipo de presa a cazar con cada uno de ellos, pero también con el soporte inicial escogido para manufacturar el cabezal, como sucede con el de Tipo A donde el largo máximo estaba limitado por el tamaño del metapodio del camélido.

\section{Tipología y tecnología del arponaje: distribución espacial y cronológica}

Los primeros habitantes del litoral desértico hace 11 mil años ya poseían un modo de vida volcado al mar y su litoral, tal como lo evidencian sus campamentos a cielo abierto en La Chimba 13 en Antofagasta y El Obispo 1 casi $350 \mathrm{~km}$ al sur, así como también sus asentamientos en aleros apostados en Paposo y Taltal (Castelleti, 2007; Cervellino et al., 2000; Llagostera 1977, 1979; Llagostera et al., 2000; Núñez y Santoro, 2011; Salazar et al., 2013, 2015). Los restos de fauna muestran que en esta época ya consumían diversas especies de moluscos, peces y algunos mamíferos marinos como otáridos y delfines, aunque aún sin desarrollar una tecnología especializada

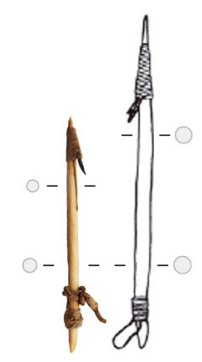

12

A

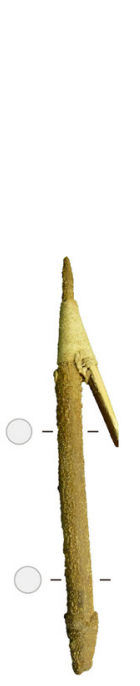

3

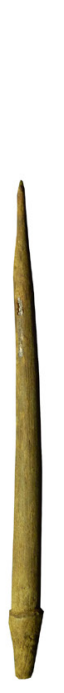

B

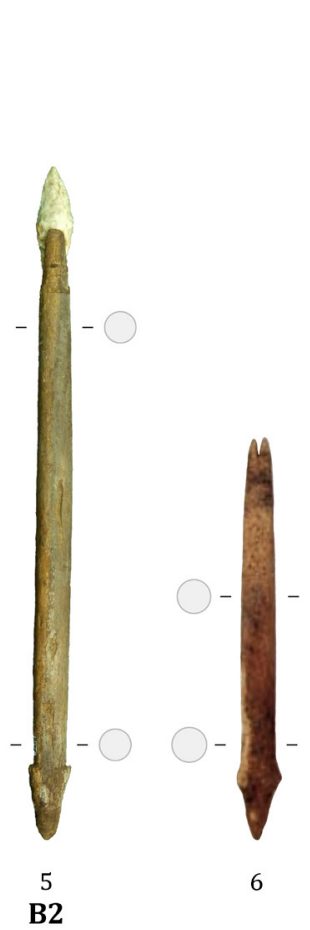

6

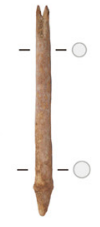

7

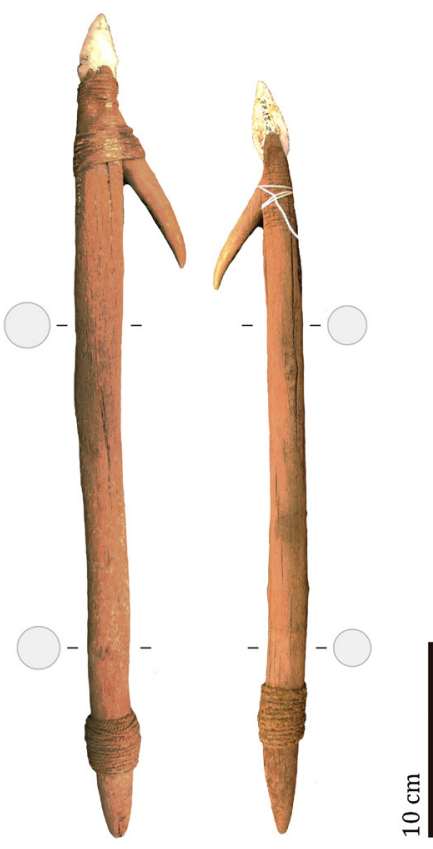

9

D

Figura 8. Tipología de arpones de la costa antofagastina. Las piezas corresponden a: (1) Las Loberas, Mejillones (MMJ); (2) Caleta Huelén, desembocadura del río Loa (Spahni, 1967: Pl.V.16); (3, 4 y 5) Punta Blanca, Tocopilla (MA); (6) Vertedero Municipal, Antofagasta (MA); (7) Michilla 02, Mejillones (Fondecyt 1110702); (8 y 9) La Chimba, Antofagasta (MQB). 


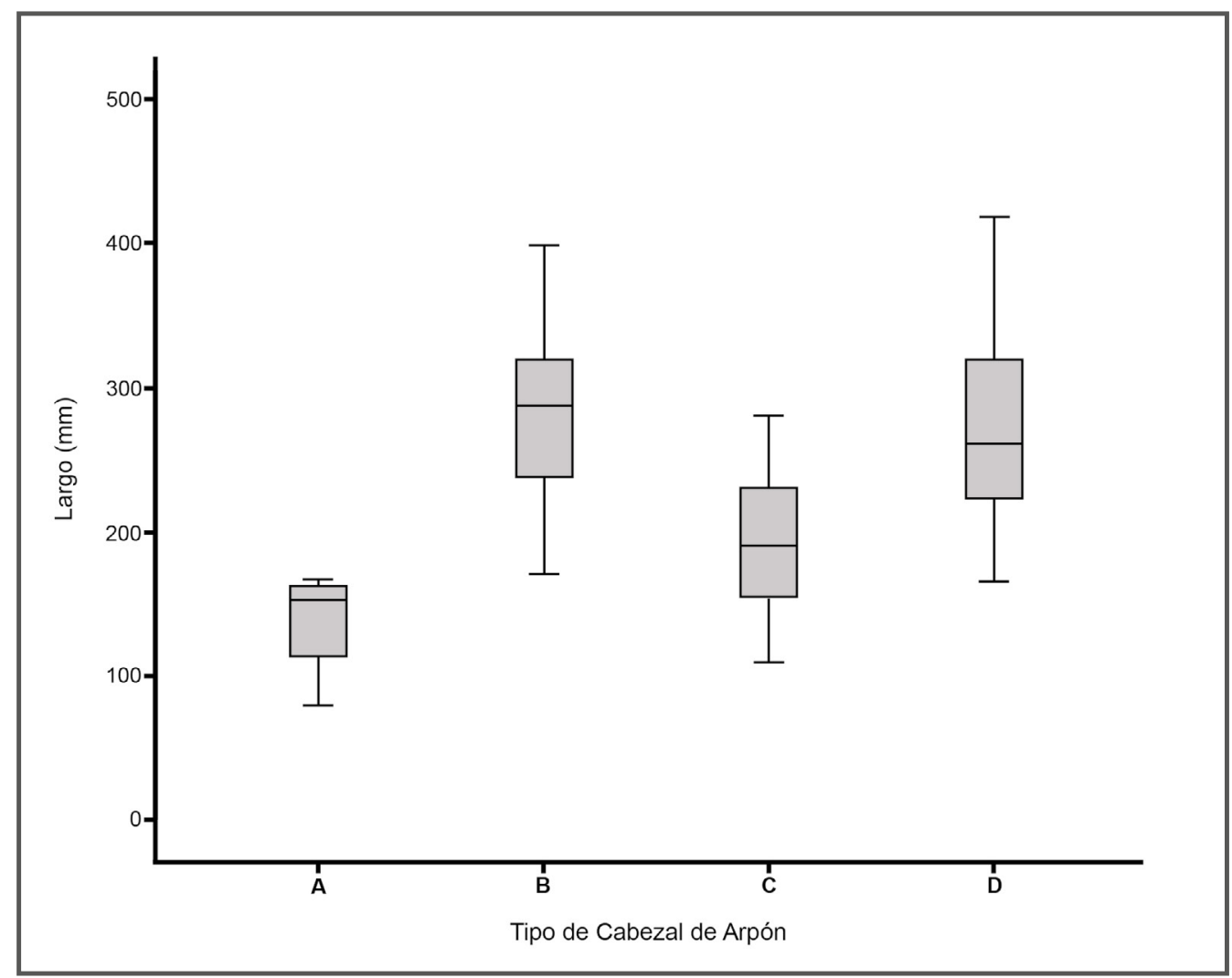

Figura 9. Relaciones métricas de los largos de cada tipo de cabezal de arpón, incluyendo mínimos, máximos, medias y varianzas (piezas completas).

en su captura. Ésta solo surge en el litoral antofagastino hacia los $7000 \mathrm{cal}$ AP debido, por un lado, a la presencia de restos de especies de alta mar en los basurales arqueológicos, como albacoras (Xiphias gladius), atunes (Thunnus sp.) y marlines (Kajikia audax), entre otras especies (Báerez et al., 2016; Castro et al., 2012; Contreras et al., 2011; Núñez et al., 1974; Olguín et al., 2014, 2015; Rebolledo et al., 2015), sincrónicamente con las primeras evidencias artefactuales de restos de arpón, especialmente barbas de hueso como aquellas características de los tipos B, C y D descritos previamente, pero también de posibles secciones de vástago, fragmentos en general poco considerados en la comprensión de los procesos de caza marina (Figura 10) (Ballester et al., 2014b, 2014c; Bird, 1943; Bittmann, 1984; Boisset et al., 1969; Castelleti, 2007; Contreras et al., 2008a, 2011; Cruz y Bravo, 1980; Montenegro, 1982; Mostny, 1964; Núñez et al., 1974; Olguín, 2011; Olguín et al., 2015; Rebolledo, 2014; Silva y
Bahamondes, 1969). Esta situación se ve corroborada además con las transformaciones en el patrón de asentamiento con el nuevo rol preponderante de las embarcaciones y la caza marina (Ballester y Gallardo, 2011; Bittmann, 1978; True, 1975).

Lamentablemente no será sino hasta siglos después, datados entre los años 6500 y 4000 cal AP, que encontramos los restos más tempranos de cabezales completos en sitios arqueológicos debido a que es cuando surgen los primeros cementerios colectivos y se vuelven populares las piezas completas en los ajuares funerarios. Augusto Capdeville, sin lugar a dudas el arqueólogo más importante de Taltal, menciona varios ejemplares en sus excavaciones en los sitios de la Civilización Dolménica correspondientes a este momento temporal (Capdeville, 1921, 1928; Mostny, 1964). Casi $450 \mathrm{~km}$ al norte en el sitio $\mathrm{CaH}$ 42, de similares características y sincrónico, fueron recuperados como ofrenda a los entierros humanos 
cabezales de arpón completos de vástago de hueso y madera, uno de estos últimos proveniente de la tumba 12 de la Estructura 1 datado directamente en 4359-3870 cal AP (Núñez, 1974; Núñez et al., 1974; Zlatar Ms. s/f 1983, 1989). De esta colección de cabezales hemos podido acceder $y$ analizar algunos del Tipo A. Si a esto agregamos la presencia de barbas de hueso en contextos basurales desde algunos siglos antes a lo largo del todo el litoral, sería a partir de este momento que la tecnología de arponaje ya estaría en pleno funcionamiento en distintas variantes, tanto el Tipo A como algunas de aquellas de mayor tamaño y potencial de retención al interior del animal, como los tipos B, C o D (Figura 10).

En los milenios posteriores, entre los 2500 y 1100 cal AP se populariza una nueva arquitectura fúnebre ahora separada del espacio residencial, caracterizada por túmulos de tierra y roca individuales donde eran depositados en una fosa central el fallecido junto a su ajuar, aumentando sustancialmente la presencia de objetos completos en las colecciones arqueológicas, aunque seriamente intervenidos por saqueadores (Ballester y Clarot, 2014; Capdeville, 1928; Gallardo et al., 2017; Latcham, 1910; Moragas, 1982; Mostny, 1964; Núñez, 1971; Spahni, 1967). Así también se popularizan los cabezales de arpón y en algunos casos fragmentos de astiles principales, siendo comunes los tipos $\mathrm{A}, \mathrm{B}$ y $\mathrm{C}$, aunque está completamente ausente el Tipo D (Figura 10). Capdeville notó hace más de 100 años algo que volvemos a corroborar con nueva evidencia y gracias a fechados radiocarbónicos: que el cabezal de arpón Tipo C solo fue utilizado durante este período, como él decía, entre "La Gente de los Túmulos de Tierra”, no encontrándolos ni antes ni después (Mostny, 1964, p. 54).

Es en este momento en que contamos con las fechas más tempranas para el cabezal Tipo B, datado directamente hacia los años 1900-1400 cal AP en el cementerio de Punta Blanca 02, mismo contexto fúnebre donde encontramos directamente asociados los Subtipos B1 y B2, haciéndolos contemporáneos. Kilómetros más al norte recuperamos piezas sincrónicas del mismo tipo en $\mathrm{CaH}$ 07, 10, 10A y 20, ampliando geográficamente su distribución (Ballester y Clarot, 2014; Gallardo et al., 2017; Núñez, 1971; Spahni, 1967). Lamentablemente los cabezales de madera recuperados de Choluto, datados varios siglos antes
(2148-1638 cal AP), se encuentran en muy malas condiciones de conservación, haciendo imposible diferenciar la técnica de retención de la línea de caza (Castelleti, 2007), aunque por el resto de sus características debiera corresponder a los tipos $\mathrm{B}$ o $\mathrm{D}$.

Quinientos ańos antes de la llegada de los europeos, entre los 1000 y los $450 \mathrm{cal} \mathrm{AP,} \mathrm{si} \mathrm{bien} \mathrm{la} \mathrm{cantidad}$ de contextos estudiados sistemáticamente es menor, no por falta de evidencia arqueológica sino al contrario por poco interés de los arqueólogos, la frecuencia de cabezales de arpón sigue siendo igual de importante que durante etapas previas (Ballester et al., 2014a; Capdeville, 1922; Chervin, 1903; Digby, 1934; Mostny, 1964; Núñez, 1971, 1987; Sénéchal de la Grange, 1903; Spahni, 1967). Ante la completa ausencia del Tipo C, surge por primera vez en la secuencia el Tipo D como predominante dentro del conjunto, lo que es claramente apreciable en sitios como Auto Club y La Chimba de Antofagasta junto a los cementerios PIT/PT de la desembocadura del Loa (CaH 02, 04, 12, 33, 51) (Figura 10) (Ballester et al., 2014a; Spahni, 1967). Esta innovación no opaca los otros dos tipos ya tradicionales siguiendo en uso las variantes A y B, recurrentes hasta en los mismos sitios arqueológicos.

El momento de contacto europeo es tal vez el menos conocido desde la arqueología. Junto a las ruinas de la antigua Caleta Loa en la desembocadura del río (Núñez, 1971) un cementerio colonial temprano (CaH 53) aún exhibe junto a sus sepulturas saqueadas fragmentos de cerámica torneada, crisoles, textiles históricos y al menos una decena de cabezales de arpón del Tipo B y unos pocos del Tipo A (Figura 10), comprobando la continuidad tecnológica y el tradicionalismo de las prácticas de caza marina de estas poblaciones incluso luego del contacto europeo.

\section{Tecnología de arponaje, modo de vida y relaciones sociales}

Donde las primeras apariencias pueden mostrar resiliencia y simplicidad, luego de miradas más profundas y detenidas no tardan en surgir los procesos, las transformaciones y la complejidad de soluciones culturales, económicas, políticas y simbólicas de un pueblo que vivió de la caza, pesca y recolección 


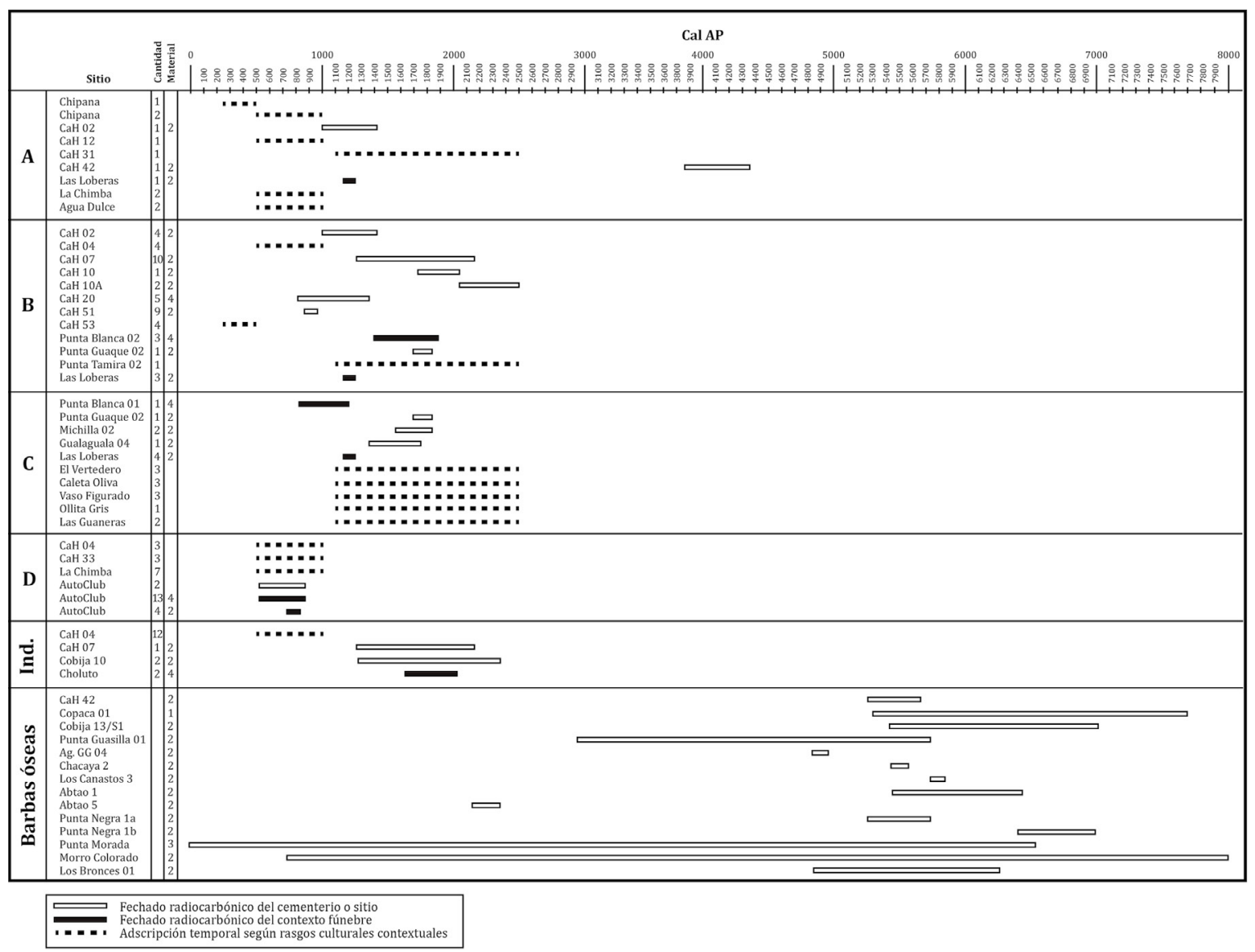

Figura 10. Distribución cronológica de los tipos de arpones y la presencia de barbas óseas de arpón (tipos $\mathrm{B}, \mathrm{C}$ o $\mathrm{D}$ ) en contextos arqueológicos. En columna material: (1) Fecha calibrada entregada en la bibliografía; (2) material terrestre calibrado mediante curva Cal13.14c (Hogg et al., 2013); (3) material marino calibrado mediante curva Marine13.14c (Reimer et al., 2013); (4) material mixto humano calibrado mediante curva Marine/So. Hem al 90\% (Hogg et al., 2013); todas calibradas en Calib 7.0.4 (Stuiver et al., 2005). $\Delta \mathrm{R}$ marino diferencial para rango 7000-4500 AP (Latorre et al., 2015) y 4000-500 AP

(Ortlieb et al., 2011).

por más de 11 mil ańos en el litoral del desierto de Atacama. No fueron siempre iguales, menos se mantuvieron impávidos ante los procesos históricos regionales y locales, del desierto y la costa. Tampoco podemos tildarlos de simples cuando crearon una tecnología altamente sofisticada en soluciones técnicas y formas de organización social. Un examen aproximativo del arponaje desde una perspectiva histórica y comparada nos permite darnos cuenta de aquello, un ejemplo entre cientos de una labor aún pendiente.

Si bien el arpón es un artefacto común en las ofrendas fúnebres, y recurrentemente mencionado en la literatura especializada, yace invisibilizado en la prehistoria y su arqueología. Poco o nada sabíamos de su mecanismo, composición, materiales, diseño, cronología, distribución geográfica, atributos técnicos y diversidad morfológica. Aliciente suficiente para emprender su estudio y sistematización no como un fetiche, sino por el enorme valor de la caza marina en la sociedad litoral. Comenzamos por aclarar gracias a la etnografía comparada de sociedades litorales la definición conceptual del arpón, y con ello también su potencialidad de uso con casos reales. Luego de su definición práctica y artefactual, gracias a dos ejercicios clasificatorios situamos la solución técnica local en la escena continental, pero también ordenamos 
por primera vez la diversidad instrumental en una clasificación tipológica de cabezales de arpón para el litoral del desierto de Atacama.

Los resultados nos permiten hablar de un dispositivo de arponaje compuesto de un astil principal largo para caza directa que no requirió de propulsor, con largas cuerdas hechas de finas tiras de cuero de lobo marino, sin boyas como es común en Norteamérica. Los cabezales mantienen una norma y diseño común, y son a lo largo de toda la secuencia "simples barbados compuestos", a diferencia de lo que ocurre en otras poblaciones como aquellas del Ártico y el Pacífico occidental (Lavondès, 1982; Leroi-Gourhan, 1945; Quimby, 1946). Una tecnología aditiva de enorme sofisticación que permitía por una parte reemplazar secciones en caso de fracturas o pérdidas sin necesidad de confeccionar desde el comienzo la pieza completa; por otra, articular un conjunto de saberes técnicos y artesanales sobre múltiples materias primas que conectaban diversos territorios, situación que nos llevará más adelante a discutir temas transversales de enorme interés cultural. La continuidad del diseño general y el modelo simple barbado compuesto a lo largo de más de 6 mil años demuestra eficiencia tecnológica, tradicionalismo artefactual y estética funcional (LeroiGourhan, 1964).

Pero tras el diseño general homogéneo y conservador se manifestaron innovaciones en variantes técnicas sobre distintos atributos del cabezal y en su combinación, especialmente en el sistema de retención del animal, modo de retención de la línea de caza, materia prima del vástago y terminación del extremo penetrante. Cuatro tipos de cabezal de arpón fueron identificados, algunos presentes en toda la secuencia temporal desde la aparición del dispositivo, otros restringidos a ciertos períodos o regiones, haciendo visibles procesos de innovación técnica y agencia tecnológica. El Tipo A, más pequeño y con barbas de reducido potencial de retención, debió destinarse a presas menores que impusieran baja resistencia y fuerza en la captura, variante que fue utilizada desde los 4400 cal AP hasta momentos posteriores al contacto europeo sin cambios en su morfología y atributos, mostrando continuidad y tradicionalismo técnico, productivo y de diseño.
Antes que eso aparecen las primeras evidencias de arpones debido a barbas de hueso y fragmentos de vástago en contextos basurales desde los $7000 \mathrm{cal}$ AP, estando completamente ausentes en los casi 5 mil ańos de ocupación litoral que le anteceden. La morfología y características de estas barbas óseas corresponden a aquellas que posteriormente veremos articuladas a los cabezales tipo $\mathrm{B}, \mathrm{C}$ o D, utilizándose como parte del dispositivo hasta momentos posteriores al contacto europeo. Estos tres tipos, al poseer tamańos superiores junto a un sistema de barba más robusta y firme, debieron estar orientados a la captura de presas mayores que ofrecieran una resistencia más dura a la actividad de caza y aprehensión.

De ellos las variantes $\mathrm{B}$ y $\mathrm{C}$ poseen sus fechados más tempranos hacia el período Formativo Litoral (2500-1100 cal AP), los primeros presentes de forma continua a lo largo de la secuencia hasta el siglo XVI, mientras que los segundos se limitan únicamente al rango comprendido para el Formativo, sin que existan ejemplares presentes posteriores a los 1100 cal AP. Pero el Tipo C además de estar restringido temporalmente no ha sido identificado a lo largo de todo el litoral, concentrándose desde el sector sur de Tocopilla en Punta Blanca hasta el sur de Taltal en Las Guaneras (Figura 5). Su ausencia en contextos más nortinos puede deberse a diversas causas, por una parte, a problemas de muestreo y falta de arqueología sistemática, por otra, producto de diferencias regionales de diseńo entre la zona septentrional y meridional del litoral antofagastino. Esta última es una opción probable si consideramos que la zona norte cuenta con una riqueza forestal notoriamente mayor que aquella del sur, en especial por los bosques de la ribera del Loa y sus oasis, como Quillagua y Chacance (60 y $70 \mathrm{~km}$ de la costa, respectivamente), pero también de la pampa del Tamarugal y el salar de Llamara (40 y $50 \mathrm{~km}$ de la costa, respectivamente), mientras que hacia el sur la merma en la disponibilidad forestal pudo fomentar el aprovechamiento de materias primas óseas de animales marinos. Si bien es una opción plausible, no explica porqué esta proliferación de cabezales con vástagos óseos de mamíferos marinos se circunscribe solo a un período particular y no continúa hacia momentos posteriores, quitando validez a la variable 
ambiental. Para ahondar en estos temas requerimos una arqueología más profunda e interregional.

El Tipo D solamente aparece en los últimos cinco siglos previos a la llegada de los europeos, volviéndolo una variante más bien tardía dentro de la secuencia. Mantiene ciertos atributos tradicionales aunque con transformaciones sectoriales importantes; el más notorio en el sistema de retención de la línea de caza reemplazando el modelado del vástago por la adición de un complemento de fibras. De todas formas esta innovación artefactual no sustituyó a su par del Tipo B; al contrario, se mantuvieron conjuntamente en uso apareciendo en algunos casos hasta en los mismos cementerios. ¿Por qué la coexistencia? Tal vez un fenómeno de renovación tecnológica que no fue de golpe sino un proceso largo donde se resguardaron soluciones tradicionales en convivencia con innovaciones, permitiéndoles funcionar paralelamente. Otra opción es que el Tipo D viniera a ocupar el lugar del desaparecido Tipo $\mathrm{C}$ en el equipo de caza, aunque sus tamaños son notoriamente distintos, volviéndolo poco probable en términos funcionales.

La existencia de normas estrictas y compartidas de diseño a más de $500 \mathrm{~km}$ de distancia fue resultado de la alta movilidad longitudinal de los pescadores y balseros tanto a nivel de capacidad de desplazamiento en largos trayectos como en su recurrencia y periodicidad, situación que debió verse reforzada gracias a lazos de parentesco y compadrazgo (Ballester y Gallardo, 2011, 2017; Ballester et al., 2010, 2015; Bittmann, 1986; Gallardo et al., 2017). Movilidad de personas que traía como consecuencia el flujo de ideas, imaginarios, conceptos, gestos y formas de hacer que gracias a relaciones sociales estrechas y habituales cuajaban en una estructura cognitiva y mental común, materializándose en una cultura tecnológica compartida que sobrepasaba a los arpones hacia todo su universo material. Basta comparar sus anzuelos, pesas, poteras, balsas de cuero de lobo marino, redes, collares, puntas de proyectil, monumentos fúnebres y estructuras habitacionales, solo por poner algunos ejemplos.

Volvamos al arpón. Luego de la síntesis de la información existente y su clasificación, podemos decir que nos enfrentamos a uno de los bienes más so- fisticados del universo material del litoral. Más allá de las variantes, constituyen artefactos compuestos de múltiples partes acopladas entre sí siguiendo un esquema preconcebido y un diseño mental de base social, con algunas secciones estáticas y rígidas en su encaje, junto a otras móviles que se articulan y ensamblan dinámicamente como parte del mecanismo de arponaje. Maquinaria compleja con módulos independientes, un engranaje de funcionamiento definido, repleta de finas sutilezas técnicas. Pero desmembremos el arpón: vástagos modelados sobre maderas densas o huesos de mamíferos marinos y terrestres, barbas óseas sobre metapodios de camélido, fibras vegetales de las cuales la más común fue el algodón, puntas líticas de rocas ricas en sílice talladas bifacialmente, delgadas líneas de caza de cuero de lobo marino, espinas de cactus recortadas y pulidas, lancetas de metal en unos pocos casos, gomas y resinas para adherir ciertas secciones, además del uso regular de pigmento rojo para mantener los cueros y cubrir los artefactos. Madera, hueso marino, hueso terrestre, cactus, metal, pigmento, lítica, algodón, fibras vegetales, resinas y cueros. El arpón condensa en un solo tipo de objeto numerosos materiales provenientes de diferentes ambientes articulados a través de cientos de actos prácticos, implicando la existencia de conocimientos de producción para las múltiples materias primas, cada una con su respectiva cadena operativa, cúmulo de saberes técnicos, herramientas de trabajo, organización laboral y distribución geográfica. Aun cuando podrían haberlos manufacturado con los materiales inmediatos a sus residencias en la costa y cercano al mar donde más tarde los utilizarían en las faenas de caza -de la misma forma que lo hicieron sin problema por ejemplo las comunidades yámana del extremo sur y ciertas parcialidades esquimales del polo norte-, por alguna razón escogieron utilizar prácticamente todos los materiales que conocían del desierto para fabricar el arpón. Cuando podrían haber aprovechado a cabalidad los recursos que entregaba el mar, más aún aquellos que obtenían mediante el mismo acto de arponaje en la caza marina como las materias primas óseas, crearon un complejo modelo clasificatorio que definía el material con que debía confeccionarse cada sección del arpón y cómo combinarse entre sí, normas productivas que bien pudieron corresponder a estrategias cognitivas y simbólicas conscientes de incorporación del desierto a su paisaje, a modo 
de un gesto ideológico de articulación territorial anclado en la tecnología. Sobre este tema puramente cultural continuaremos trabajando en futuras investigaciones, ya que no es aquí ni ahora el lugar ni el momento.

Ahora vayamos al cazador. En la introducción nos guardamos a propósito para el final el desenlace del relato de Vázquez de Espinoza (1948 [1630], p. 619) sobre la caza de la ballena:

...el indio vuelve a cobrar su babilla, y se viene a tierra a ojear, y atalayar adonde viene a morir a la costa, y así están en centinela, hasta que la ven parar donde va luego toda aquella parcialidad, y parentela, que ha estado con cuidado a la mirar, juntos todos con los amigos, y vecinos para el convite, la abren por un costado, donde están comiendo unos dentro, y otros fuera seis y a ocho días hasta que de hedor no pueden estar allí, en este tiempo hinchen todas sus vasijas (que las mas son de tripas de lobo marino) de lonjas de la vallena.

Si bien la práctica de la caza fue una actividad especializada y restringida a ciertos individuos del grupo, aquellos conocedores del arte del arponaje y el navegar, el acto de consumo de los enormes volúmenes de carne, grasas, huesos y pieles extraídas fue colectivo, sobrepasando las unidades domésticas de los cazadores, un momento importante de congregación social. Fuera a la manera de grandes festines comunales o para acumular el excedente pensando

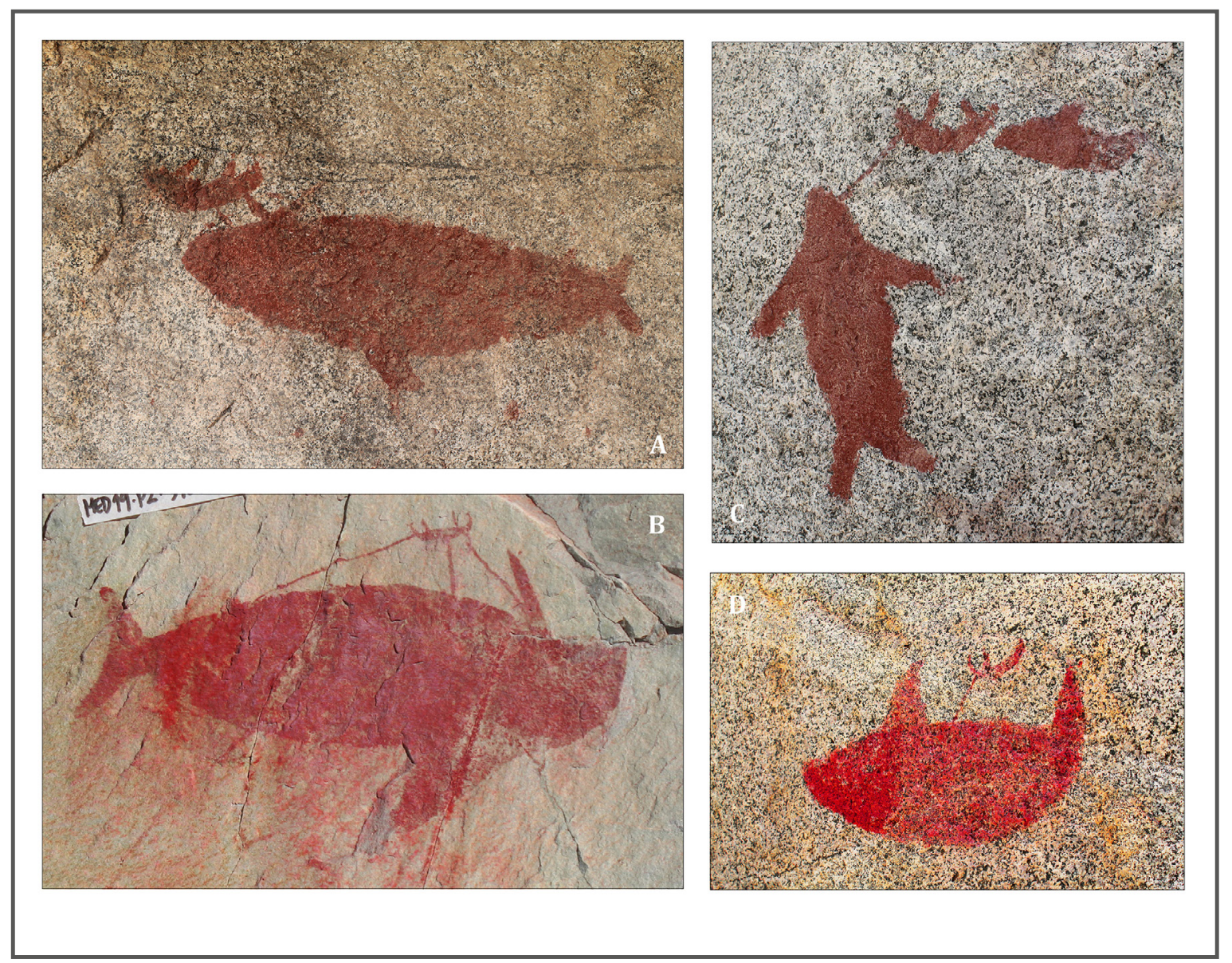

Figura 11. Escenas de caza de grandes presas marinas mediante arponeo en el arte rupestre El Médano: (A) Quebrada de Izcuña, I-02, B-08, P-1; (B) Quebrada El Médano, Md-19, P-2; (C) Quebrada de Izcuńa, I-09, B-9, P-1; y (D) Quebrada de Izcuña, I-09, B-2, P-1. (Fotografía B de Francisco Gallardo; Fotografías A, C, D del autor). 
en el futuro para un consumo diferido, la labor de los cazadores era en beneficio del grupo completo (Hayden, 2014). Este gesto de distribución colectiva ponía a los balseros y cazadores en una situación diferencial frente a sus pares, desequilibrando la balanza económica de la estructura organizacional al poner a unos pocos actores portadores de un conocimiento experto encargados de una labor específica en una condición política y social privilegiada (Earle y Springgs, 2015; Godelier y Strathern, 1991; Hirth, 1996; Spielmann, 2002; Testart, 1988). Una estructura social modelada por la concentración de conocimiento, la división laboral y las relaciones de distribución. Tal vez sobre este mismo desequilibrio económico y político se ancla el motivo tras el cual en sus representaciones rupestres los balseros y la caza marina toman papeles protagónicos que invisibilizan ideológicamente las otras cientos de prácticas, actores y roles que definen el modo de vida litoral (Ballester, 2018) (Figura 11).

El funcionamiento y reproducción de esta estructura social dependía de que la caza fuera fructífera, condición en la cual las embarcaciones y el arponaje eran fundamentales. Pilar material que además era uno de sus artefactos más sofisticados y complejos. Para utilizarlos había que recorrer previamente cientos de kilómetros de desierto no sin antes conocerlo a cabalidad, poseer múltiples saberes técnicos sobre cada materia prima y sus cualidades, además de disponer herramientas para cada trabajo específico y más importante aún, disponer del tiempo para realizarlo sin poner en jaque la reproducción de sus unidades domésticas. Todo esto a orillas de uno de los océanos más ricos y productivos del mundo, donde las comunidades habitaban prácticamente en la opulencia, viviendo sin necesidad de la caza marina con arponaje y embarcaciones por más de 5 mil años. Mi pregunta final: ¿¿El arpón solo servía para apaciguar el hambre del salvaje pueblo costero? Evidentemente no.

\section{Agradecimientos}

FONDECYT 1110702 y 1160045 , FONDAP 15110006. A las instituciones Museo de Antofagasta (MA), Museo Augusto Capdeville Rojas de Taltal (MACRT), Museo de Mejillones (MMJ), Univer- sidad de Antofagasta (UA), Museo Arqueológico San Miguel de Azapa de la Universidad de Tarapacá (MASMA), American Museum of Natural History de Nueva York (AMNH) y al Musée du Quai Branly de París (MQB). A Francisco Gallardo, Alexander San Francisco, José Blanco, Alejandro Clarot, Daniel Quiroz, Patricio de Souza y Marianne Christensen por sus comentarios y recomendaciones. Finalmente a Carolina Agüero y Marcela Aguirre por su trabajo editorial.

\section{Referencias citadas}

Acosta, J. (1590). Historia natural y moral de las Indias: en que se tratan las cosas notables del cielo y elementos, metales, plantas, y animales dellas y los ritos, y ceremonias, leyes $y$ govierno, y guerras de los indios. Sevilla: Casa de Juan de León.

Arima, E. (1988). Notes on Nootkan sea mammal hunting. Arctic Anthropology, 25(1), 16-27.

Arnold, C. (1989). Artic harpoons. Artic, 42(1), 80-81.

Arnold, J. (1995). Transportation innovation and social complexity among maritime hunter-gatherers societies. American Anthropology, 97(4), 733-747.

Arnold, J. y Bernard, J. (2005). Negotiating the coasts: status and the evolution of boat technology in California. World Archaeology, 37(1), 109-131.

Arnold, J., Colten, R. y Pletka, S. (1997). Contexts of cultural change in insular California. American Antiquity, 62(2), 300-318.

Ballester, B. (2017). La delgada línea roja: sogas de arpón de los últimos cazadores marinos del norte de Chile (10001500 DC). Revista Chilena de Antropología, 35, 47-71.

Ballester, B. (2018). El Médano rock art style: Izcuña paintings and the marine hunter-gatherers of the Atacama Desert. Antiquity, 92(361), 132-148.

Ballester, B. y Álvarez, J. (2014-2015). Nadando entre alegorías tribales o la crónica del descubrimiento de las pinturas de Izcuña. Taltalia, 7-8, 9-17.

Ballester, B. y Clarot, A. (2014). La gente de los túmulos de tierra. Santiago: Marmot Impresores. 
Ballester, B. y Gallardo, F. (2011). Prehistoric and historic networks on the Atacama Desert coast (northern Chile). Antiquity, 85, 329, 875-889.

Ballester, B. y Gallardo, F. (2016). Painting a lost world. The red rock art of El Médano. Current World Archaeo$\log y, 77,36-38$.

Ballester, B. y Gallardo, F. (2017). La versatilidad del parentesco en la reproducción social: El caso de los cazadores-pescadores marinos del desierto de Atacama (siglos XVI-XIX, norte de Chile). Boletín de la Sociedad Chilena de Arqueología, 47, 7-28.

Ballester, B., San Francisco, A. y Gallardo, F. (2010). Modo de vida y economía doméstica de las comunidades cazadoras recolectoras costeras del desierto de Atacama durante tiempos coloniales y republicanos. Taltalia, 3 , 21-32.

Ballester, B., Clarot, A. y Llagostera A. (2014a). El Cementerio de Auto Club de Antofagasta y la sociedad litoral entre los 1000 y 1450 d.C. Hombre y Desierto, 18, 187212.

Ballester, B., Clarot, A., Bustos, V., Llagostera, A. y Garcés, H. (2014b). Arqueología de la prehistoria de la Península de Mejillones: el campamento de Los Canastos 3 desde sus cuadernos de campo y materiales de museo. Boletín de la Sociedad Chilena de Arqueología, 43/44, 5-21.

Ballester, B., Clarot, A. y Bustos, V. (2014c). Chacaya 2: reevaluación de un campamento Arcaico Tardío (6000 al 4000 cal. AP) de la costa de Mejillones, II Región, Chile. Werkén, 15, 31-48.

Ballester, B., Gallardo, F. y Aguilera, P. (2015). Representaciones que navegan más allá de sus aguas: una pintura estilo El Médano a más de $250 \mathrm{~km}$ de su sitio homónimo. Boletín de la Sociedad Chilena de Arqueología, 45, 81-94.

Ballester, B., Calás, E., Peregrino, C., Vidal, E. y Aguilera, P. (2017). La vida en comunidad de los cazadorespescadores marinos del desierto de Atacama (4000-2000 cal. a.C.). En Gallardo, F., Ballester, B. y Fuenzalida, N. (Eds.). Monumentos funerarios de la costa del desierto de Atacama. Los cazadores-recolectores marinos y sus intercambios (500 a.C.-700 d.C.) (pp. 183-197). Santiago: CIIR \& SCHA.
Barraza, A. (1981). Descripción, análisis y funcionalidad. Colección Capdeville Taltal-Chile. Memoria para optar al título de Arqueólogo, Universidad del Norte sede Antofagasta.

Béarez, P., Fuentes-Muchen, F., Rebolledo, S., Salazar, D. y Olguín, L. (2016). Billfish foraging along the northern coast of Chile during the Middle Holocene (7400-5900 cal BP). Journal of Anthropological Archaeology, 41, 185195.

Beauchamp, W. (1902). Horn and bone implements of the New York Indians. Bulletin of the University of the State of New York, 50, 243-350.

Bennyhoff, J. (1950). Californian fish spears and harpoons. Anthropological Records, 9(4), 295-337.

Berenguer, J. (2009). Las pinturas de El Médano, norte de Chile: 25 años después de Mostny y Niemeyer. Boletín del Museo Chileno de Arte Precolombino, 14(2), 57-95.

Bernard, J. (2004). Status and the swordfish: the origins of large-species fishing among the Chumash. En Arnold, J. (Ed.). Foundations of humash complexity (pp. 25-51). Los Angeles, CA: Cotsen Institute of Archaeology, University of California.

Bethune, J. (1848 [1622]). The observations of Sir Richard Hawkins, Knt in his voyage into the South Sea in the year 1593. London: Printed for The Hawlyut Society.

Bibar, G. (1966 [1558]). Crónica y relación copiosa y verdadera de los Reynos de Chile. Santiago: Fondo Histórico y Bibliográfico José Toribio Medina, Dibam.

Bird, J. (1943). Excavations in northern Chile. Anthropological Papers of the American Museum of Natural History, 38(4), 173-318.

Bird, J. (1946). The cultural sequence of the north Chilean coast. Bulletin of the Bureau of American Ethnology, 143, 587-594.

Birket-Smith, K. (1953). Los esquimales. Barcelona: Labor.

Bittmann, B. (1978). Fishermen, mummies and balsa rafts on the coast of northern Chile. El Dorado, III(3), 60-103. 
Bittmann, B. (1984). El proyecto Cobija: investigaciones antropológicas en la costa del Desierto de Atacama (Chile). En XLIV Congreso Internacional de Americanistas, Simposio Culturas Atacameñas (pp. 99-146). Manchester.

Bittmann, B. (1986). Los pescadores, cazadores y recolectores de la costa árida chilena: un modelo arqueológico. Chungara, 16-17, 59-65.

Bittmann, B. y Munizaga, J. (1983). Comments on a double mummy containing a spear thrower in the "Anker Nielsen Collection", Iquique, northern Chile. Indiana, 9, 383-419.

Black, G. (1890). Notice of a peculiar type of implement found in ancient graves in Peru. Proc. Soc. Antiq. Scot., 23, 164-166.

Boas, F. (1888). The Central Eskimo. Annual Report of the Bureau of Ethnology of the Secretary of the Smithsonian Institution, 6, 409-669.

Boas, F. (1907). Second report on the Eskimo of Baffin land and Hudson Bay. Bulletin of the American Museum of Natural History, 15(2), 374-570.

Bockstoce, J. (1976). On the development of whaling in the western Thule Culture. Folk, 18, 41-46.

Boisset, G., Llagostera, A. y Salas, E. (1969). Excavaciones arqueológicas en Caleta Abtao. Antofagasta. En Actas del $V$ Congreso Nacional de Arqueología (pp. 75-152). La Serena: Dibam.

Bollaert, W. (1854). Observations on the History of the incas of Peru, on the indians of South Peru, and on some indian remains in the Province of Tarapacá. Journal of the Ethnological Society of London, 3, 132-164.

Bollaert, W. (1860). Antiquarian, ethnological and other research in New Granada, Ecuador, Peru and Chile, with observations of the Prelncarial, Incarial and other monuments of Peruvian Nations. London: Trubner and Co.

Bravo, L. (1981). Abtao-5: un modelo de adaptación tardia a la costa de la Segunda Región. Memoria para optar al título de Arqueólogo, Universidad del Norte sede Antofagasta.

Bridges, T. (1875). Tierra del Fuego, Ushuwia. South American Missionary Magazine, 1, 5-16.
Brown, L. (1967). Toggle head harpoons of the Central Plains. Plains Anthropologist, 12(38), 356-362.

Capdeville, A. (1921). Notas acerca de la arqueología de Taltal. II Civilización dolménica. Boletín de la Academia Nacional de Historia, 3(5), 256-261.

Capdeville, A. (1922). Notas acerca de la arqueología de Taltal. III Civilización de las gentes de los vasos pintados. Boletín de la Academia Nacional de Historia, 3, 7-8, 1-9.

Capdeville, A. (1928). Cómo descubrí la industria paleolítica americana de los sílices negros tallados, en zona de la costa de Taltal. Revista Chilena de Historia Natural, 32(1), 348-364.

Casassas, J. (1992). Carta del Factor de Potosí Juan Lozano Machuca (al virrey del Perú Don Martín Enríquez) en que da cuenta de cosas de aquella villa y de las minas de los Lipes (año 1581). Estudios Atacameños, 10, 30-34.

Castelleti, J. (2007). Patrón de asentamiento y uso de recursos a través de la secuencia ocupacional prehispánica en la costa de Taltal. Tesis para optar al grado de Magíster en Antropología con mención en Arqueología, Universidad Católica del Norte.

Castro, V., Aldunate, C. y Varela, V. (2012). Paisajes culturales de Cobija, costa de Antofagasta, Chile. Revista Chilena de Antropología, 26(2), 97-128.

Caulfielf, R. (1993). Aboriginal subsistence whaling in Greenland: the case of eqertarsuaq Municipality in West Greenland. Arctic, 46(2), 144-155.

Cavanagh, D. (1983) Ms. Northwest Coast whaling: a new perspective. Unpublished Master of Arts Thesis, University of British Columbia, Vancouver.

Cervellino, M., Llagostera, A., Weisner, R. y Castillo, G. (2000). El Obispo 1: primeras evidencias del Arcaico Temprano en la costa de la Región de Atacama. En Actas del XIV Congreso Nacional de Arqueología Chilena (pp. 483-500). Museo Regional de Atacama, Copiapó.

Chervin, A. (1902). Cranes, pointes de flèches en silex et instruments de peche provenants de la baie d'Antofagasta. Bulletin et Mémoires de la Societé d'Anthropologie de Paris, 5(3), 700-708. 
Collins, H. (1941). Prehistoric Eskimo harpoon heads from Bering Strait. Journal of the Washington Academy of Sciences, 31(7), 318-324.

Colten, R. y Arnold, J. (1998). Prehistoric marine mammal hunting on California's Northern Channel islands. American Antiquity, 63(4), 679-701.

Contreras, R. y Núñez, P. (2009). Nuevos antecedentes sobre la balsa de cuero de lobo en la costa de Taltal, Chile. Taltalia, 2, 88-97.

Contreras, R., Cruz, J., Garcés, H., Llagostera, A., Núñez, P., Rodríguez, O., Becerra, G. y Gárate, H. (2008a). Los Bronces-1: un asentamiento de 5500 años en la costa de Taltal. Taltalia, 1, 61-74.

Contreras, R., Núñez, P. y Rodríguez, O. (2008b). El Médano: reflexiones antropológicas en torno a la cosmovisión de los habitantes prehispánicos de la costa sur del Norte Grande. Taltalia, 1, 87-122.

Contreras, R., Núñez, P., Llagostera, A., Cruz, J., San Francisco, A., Ballester, B., Rodríguez, O. y Becerra, G. (2011). Un conglomerado del período Arcaico costero Medio del área Taltal Paposo, norte de Chile. Taltalia, 4, 7-31.

Coté, C. (2010). Spirits of our whaling ancestors. Seattle: University of Washington Press.

Cruz, J. y Bravo, L. (1980). Cobija Sur-1, sitio de ocupación temprana en la costa de Cobija, II Región, Chile. En Bittmann, B. (Ed.). Cobija: proyecto de investigaciones interdisciplinarias en la costa Centro Sur Andina (Chile) (pp. 153-174). Universidad del Norte, Antofagasta.

Davenport, D., Johnson, J. y Timbrook, J. (1993). The Chumash and the swordfish. Antiquity, 67, 257-272.

Digby, A. (1934). An interesting survival in harpoon design from Chile. Man, 34, 125

D’Orbigny, A. (1945) [1847]. Viaje a la América Meridional, Brasil, República de Uruguay, República Argentina, La Patagonia, República de Chile, República de Bolivia, República de Perú, realizado de 1826 a 1833. Buenos Aires: Editorial Futuro.

Earle, T. y Spriggs, M. (2015). Political economy in prehistory: a marxist approach to Pacific sequences. Current Anthropology, 56(4), 515-544.
Ewbank, T. (1855). A description of the Indians antiquities brought from Chile and Peru, by the U.S. Naval Astronomical Expedition. En Gilliss, J. (Ed.). U.S. Naval Astronomical Expedition to the Southern Hemisphere during the Years 1849, 50, 51, 52, vol. 2 (pp. 111-150). Washington DC: Nicholson Printer.

Feuillée, L. (1714). Journal des observations physiques, mathématiques, et botaniques faites par l'ordre du Roy sur les côtes orientales de l'Amérique Méridionale, et Dans les indes occidentales, depuis l'année 1707, jusques en 1712. Paris: Chez Pierre Giffart, libraire, Graveur du Roy \& de l'Académie Royale de Peinture \& de Sculpture.

Figueroa, V., Salazar, D., Mille, B. y Manríquez, G. (2015). Metal use and production among coastal societies of the Atacama Desert. Archaeometry, 57(4), 687-703.

Focacci, G. (1974). Excavaciones en el cementerio de Playa Miller 7. Arica (Chile). Chungara, 3, 23-74.

Focacci, G. y Chacón, S. (1989). Excavaciones arqueológicas en los faldeos del Morro de Arica. Sitios Morro 1/6 y 2/2. Chungara, 22, 15-62.

Gallardo, F., Cabello, G., Pimentel, G., Sepúlveda, M. y Cornejo, L. (2012). Flujos de información visual, interacción social y pinturas rupestres en el desierto de Atacama (norte de Chile). Estudios Atacameños, Arqueología y Antropologia Surandinas, 43, 35-52.

Gallardo, F., Ballester, B. y Fuenzalida, N. (2017). Monumentos funerarios de la costa del desierto de Atacama. Los cazadores-recolectores marinos y sus intercambios (500 a.C.700 d.C.). Santiago: CIIR \& SCHA.

Godelier, M. y Strathern, M. (1991). Big men and great men. Personifications of power in Melanesia. Wiltshire: Maison des Sciences de I'Homme and Cambridge University Press.

Gould, R. (1970). Spears and spears-throwers of the western desert aborigines of Australia. American Museum Novitates, 2403, 1-42.

Grier, C. (1999). The organization of production in prehistoric Thule whaling societies of the Central Canadian Arctic. Journal Canadien d'Archaéologie, 23, 11-28.

Gudger, E. (1942). Swordfishing with the harpoon in New England waters. I. The Scientific Monthly, 54(5), 418-430. 
Harkin, M. (1998). Whales, chiefs, and giants: an exploration into Nuu-Chah-Nulth political thought. Ethnology, 37(4), 317-332.

Hayden, B. (2014). The power offeasts. From prehistory to the present. New York: Cambridge University Press.

Heizer, R. (1943). A Pacific Eskimo invention in whale hunting in historic times. American Anthropologist, 45(1), 120-122.

Heizer, R. (1974). Were the Chumash whale hunters? Implications for ethnography in 1974. The Journal of California Anthropology, 1(1), 26-32.

Hirth, K. (1996). Political economy and archaeology: perspectives on exchange and production. Journal of Archaeological Research, 4(3), 203-239.

Hogg, A., Hua, Q., Blackwell, P., Niu, M., Buck, C., Guilderson, T., Heaton, T., Palmer, J., Reimer, P., Reimer, R., Turney, C. y Zimmerman, S. (2013). SHCal13 Southern Hemisphere calibration, 0-50,000 years cal BP. Radiocarbon, 55(4), 1889-1903.

Hoover, R. (1974). Some observations on Chumash prehistoric stone effigies. Journal of California Anthropology, 1(1), 33-40.

Hudson, T. y Conti, K. (1981). The "Aquatic Motif" in Chumash rock art. Journal of California and Great Basin Anthropology, 3(2), 224-231.

Huelsbeck, D. (1988). Whaling in the precontact economy of the Central Northwest Coast. Arctic Anthropology, 25(1), 1-15.

Hyades, P. (1885). La Chasse et la pêche chez les fuéguins de l'archipel du Cap Horn. Revue d'Ethnographie, 4, 514-553.

Jensen, A. (2012). The material culture of Inupiat whaling: an ethnographic and ethnohistorical perspective. Arctic Anthropology, 49(2), 143-161.

Julien, M. (1982). Les harpons Magdaléniens. Paris: Éditions du CNRS.

Krieger, H. (1926a). The collection of primitive weapons and armor of the Philippine Islands in the United States National Museum. Bulletin of the Smithsonian Institution of the United States National Museum, 137, 1-128.
Krieger, H. (1926b). Material culture of the people of southeastern Panamá, based on specimens in the United States National Museum. Bulletin of the Smithsonian Institution of the United States National Museum, 134, 1-141.

Krupnik, I. (1987). The bowhead vs. the gray whale in Chukotkan aboriginal whaling. Artic, 40(1), 16-32.

Krupnik, I. y Kan, S. (1993). Prehistoric Eskimo whaling in the Arctic: slaughter of calves or fortuitous ecology? Artic Anthropology, 30(1), 1-12.

Labarca, R., Calás, E. y Prieto, A. (2017). Los artefactos óseos de contextos funerarios costero de la región de Antofagasta. En Gallardo, F., Ballester, B. y Fuenzalida, N. (Eds.). Monumentos funerarios de la costa del desierto de Atacama. Los cazadores-recolectores marinos y sus intercambios (500 a.C.-700 d.C.) (pp. 95-106). Santiago: CIIR \& SCHA.

Lantis, M. (1938). The Alaskan whale cult and its affinities. American Anthropologist, 40, 438-464.

Larsen, H. y Rainey, F. (1948). Ipiutak and the artic whale hunting culture. Anthropological Papers of the American Museum of Natural History, 42, 1-276.

Latcham, R. (1910). Los changos de las costas de Chile. Imprenta Cervantes.

Latcham, R. (1938). Arqueología de la Región Atacameña. Santiago: Prensas de la Universidad de Chile.

Lavondès, A. (1982). Note sur les harpons polynésiens. Journal de la Société des Océanistes, 74-75(38), 143-155.

Leroi-Gourhan, A. (1935). Le kayak et le harpon des Eskimos. La Nature, 63, 510-512.

Leroi-Gourhan, A. (1946). Archéologie du Pacifique Nord. Travaux et Mémoires de l'Institut d'Ethnologie, 47, 1-542.

Leroi-Gourhan, A. (1964). Le geste et la parole. Paris: Éditions Albin Michel.

Leroi-Gourhan, A. (1973). Évolution et techniques. Milieu et techniques. Paris: Albin-Michel.

Lizárraga, R. (1999) [1603-1609]. Descripción del Perú, Tucumán, Río de la Plata y Chile. Buenos Aires: Union Académique Internationale, Academia Nacional de Historia. 
Llagostera, A. (1977). Ocupación humana en la costa norte de Chile asociada a peces locales-extintos y a litos geomé-

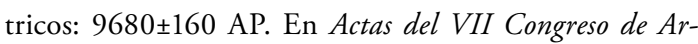
queología Chilena (pp. 93-113). Altos de Vilches.

Llagostera, A. (1979). 9700 years of maritime subsistence on the pacific: an analysis by means of bioindicators in the North of Chile. American Antiquity, 44(2), 309-324.

Llagostera, A. (1989). Caza y pesca marítima. En Hidalgo, J., Schiappacasse, V., Niemeyer, H., Aldunate, C. y Solimano, I. (Eds.). Prehistoria. Desde sus origenes hasta los albores de la conquista (pp. 57-81). Santiago: Andrés Bello.

Llagostera, A. (1990). La navegación prehispánica en el norte de Chile: bioindicadores e inferencias teóricas. Chungara, 24/25, 37-51.

Llagostera, A., Weisner, R., Castillo, G., Cervellino, M. y Costa-Junqueira, M. (2000). El Complejo Huentelauquén bajo una perspectiva macroespacial y multidisciplinaria. En Actas del XIV Congreso Nacional de Arqueología Chilena (pp. 461-480). Museo Regional de Atacama, Copiapó.

Losey, R. y Yang, D. (2007). Opportunistic whale hunting on the southern northwest coast: ancient DNA, artifact, and ethnographic evidence. American Antiquity, 72(4), 657-676.

Lothrop, S. (1928). The indians of Tierra del Fuego. Contributions from the Museum of the American Indian Heye Fundation, 10, 1-244.

Märgärit, M., Popovici, D. y Vlad, F. (2010). L'utilisation du harpon dans la culture Gumelnița. Etude de cas: l'habitat de Borduşani-Popină (Departement de Ialomița). Cercetări Arheologice, 17, 35-54.

Martial, L., Deniker, J. y Hyades, P. (2007). Etnografía de los indios yaghan en la misión cientifica del Cabo de Hornos 1882-1883. Legoupil, D. y Prieto, A. (Eds.). Punta Arenas: Ediciones Universidad de Magallanes e Instituto Francés de Estudios Andinos.

Mason, O. (1893). Throwing-sticks from Mexico and California. Proceedings of the United States National Museum, 16, 219-221.
Mason, O. (1902). Aboriginal American harpoons. A study in ethnic distribution and invention. Report of the United States National Museum, 1900, 189-304.

Mauss, M. (1906). Essai sur les variations saisonnières des sociétés Eskimo. Étude de morphologie sociale. Année Sociologique, 9, 39-132.

McCartney, A. (1980). The nature of Thule Eskimo whale use. Arctic, 33(3), 517-541.

McCartney, A. y Savelle, J. (1985). Thule Eskimo whaling in the Central Canadian Arctic. Arctic Anthropology, 22(2), 37-58.

Meggison, M. (2000). Through thick and thin: a regional comparison of harpoon heads from Thule sites in Nunavut, Canada. Thesis Submitted to the School of Graduate Studies in Master of Arts, McMaster University. Canada.

Meighan, C. (2000). Rock art on the Channel Islands of California. Pacific Coast Archaeological Society Quarterly, $36(2), 15-29$.

Monks, G., McMillan, A. y Claire, D. (2001). Nuu-Chah-Nulth whaling: archaeological insights into antiquity, species preferences and cultural importance. Artic Anthropology, 38(1), 60-81.

Montenegro, N. (1982). Sitio Punta Guasilla 1, un aporte a la arqueología del norte desértico de Chile. Memoria para optar al título de Arqueóloga, Universidad del Norte, Antofagasta.

Moragas, C. (1982). Túmulos funerarios en la costa sur de Tocopilla (Cobija), II Región. Chungara, 9, 152-173.

Mostny, G. (1942). Informe preliminar sobre las excavaciones efectuadas en la costa chilena entre Pisagua y Coquimbo del 8 de Octubre de 1941 al 15 de Marzo de 1942. Boletín del Museo Nacional de Historia Natural, 20, 97-102.

Mostny, G. (1964). Arqueología de Taltal. Epistolario de Augusto Capdeville con Max Uhle y otros arqueólogos e historiadores. Santiago: Fondo Histórico y Bibliográfico José Toribio Medina.

Mostney, G. y Niemeyer, H. (1983). Arte rupestre chileno. Santiago: Ministerio de Educación, Serie el Patrimonio Cultural Chileno. 
Mostny, G. y Niemeyer, H. (1984). Arte rupestre en El Médano, II Región. Creces, 9(5), 2-5.

Mulville, J. (2002). The role of Cetacea in prehistoric and historic Atlantic Scotland. International Journal of Osteoarchaeology, 12, 34-48.

Nelson, E. (1900). The Eskimo about Bering Strait. Washington: Government printing office.

Niemeyer, H. (2010). Crónica de un descubrimiento. Las pinturas rupestres de El Médano, Taltal. Santiago: Museo Chileno de Arte Precolombino.

Núñez, L. (1962). Contactos culturales prehispánicos entre la costa y la subcordillera andina. Boletín de la Universidad de Chile, 31, 42-47.

Núñez, L. (1971). Secuencia y cambio en los asentamientos humanos de la desembocadura del río Loa, en el norte de Chile. Boletin de la Universidad de Chile, 112, 2-25.

Núñez, L. (1987). Tráfico de metales en el área centro-sur andina: factos y expectativas. Cuadernos Instituto Nacional de Antropología, 12, 73-105.

Núñez, L. (1999). Archaic adaptation on the South-Central Andean Coast. En Blake, M. (Ed.). Pacific Latin American in prehistory. The evolution of Archaic and Formative cultures (pp.199-211). Washington D.C.: Washington State University Press.

Núñez, L. (2006). Asentamientos formativos complejos en el centro-sur andino: cuando la periferia se constituye en núcleo. Boletín de Arqueología PUCP, 10, 321-356.

Núñez, L. y Santoro, C. (2011). El tránsito Arcaico-Formativo en la circumpuna y valles occidentales del Centro Sur Andino: hacia los cambios "neolíticos". Chungara, Revista de Antropología Chilena, 43(1), 487-530.

Núñez, L., Zlatar, V. y Núñez, P. (1974). Caleta Huelén 42: una aldea temprana en el norte de Chile (nota preliminar). Hombre y Cultura, 2(5), 67-103.

Núñez, P. (1974). Notas sobre la aldea preagrícola Caleta Huelén 42, desembocadura del río Loa. Norte de Chile. Serie Documentos de Trabajo, 5, 27-43.

Núñez, P. y Contreras, R. (2003). Pinturas prehispánicas de Taltal. Fondart, Antofagasta.
Núñez, P. y Contreras, R. (2006). El arte rupestre de Taltal, norte de Chile. En Actas del V Congreso Chileno de Antropología, vol. 1 (pp. 348-357). San Felipe.

Núñez, P. y Contreras, R. (2008). El arte rupestre de Taltal norte de Chile. Taltalia, 1, 77-85.

Olguín, L. (2011). Procesos de formación y secuencia ocupacional del sitio Agua Dulce en la costa arreica del desierto de Atacama, Provincia de Taltal, Región de Antofagasta. Memoria para optar al título de Arqueóloga, Universidad de Chile, Santiago.

Olguín, L., Salazar, D. y Jackson, D. (2014). Tempranas evidencias de navegación y caza de especies oceánicas en la costa pacífica de Sudamérica (Taltal, -7.000 años cal. AP). Chungara. Revista de Antropología Chilena, 46(2), 177-192.

Olguín, L., Castro, V., Castro, P., Peña-Villalobos, I., Ruz, J. y Santander, B. (2015). Exploitation of faunal resources by marine hunter-gatherer groups during the Middle Holocene at the Copaca 1 site, Atacama Desert coast. Quaternary International, 373, 4-16.

Ortlieb, L., Vargas, G. y Saliège, J. (2011). Marine radiocarbon reservoir effect along the northern Chile-southern Peru coast $\left(14-24^{\circ} \mathrm{S}\right)$ throughout the Holocene. Quaternary Research, 75, 91-103.

Owen, B. (1998). Bows and spearthrowers in Southern Peru and Northern Chile: evidence, dating, and why it matters. Paper presented at the $63^{\text {rd }}$ Annual Meeting of the Society for American Archaeology, Seattle.

Park, R. y Mousseau, P. (2003). How small is too small? Dorset Culture "miniature" harpoon heads. Canadian Journal of Archaeology, 27, 258-272.

Paton, K. y Savelle, J. (2006). The symbolic dimensions of whale bone use in Thule winter dwellings. Études/Inuit/ Studies, 30(2), 137-161.

Pernoud, R. (1990). América del Sur en el siglo XVIII. Misceláneas anecdóticas y bibliográficas. México: FCE.

Pétillon, J. (2008). Des barbelures pour quoi faire? Réflexions préliminaires sur la fonction des pointes barbelées du Magdalénien Supérieur. Palethnologie, 1, 69-102. 
Petitot, É. (1887). Les grands esquimaux. Paris: Librairie Plon.

Porcasi, J. y Fujita, H. (2000). The dolphin hunters: a specialized prehistoric maritime adaptation in the Southern California Channel islands and Baja California. American Antiquity, 65(3), 543-566.

Pretty, F. (1904 [1599]). The prosperous voyage of M. Thomas Candish esquire into the South sea, and so round about the circumference of the whole earth, begun in the yere 1586 and finished 1588. En Hakluyt, R. (Ed.). The principal navigations, voyages, traffiques \& discoveries of the English nation made by sea or over-land to the remote and farthest distant quarters of the earth at an time within the compasse of these 1600 yeeres, vol. 11 (pp. 290-347). Glasgow: Printed at The University Press.

Quimby, G. (1946). Toggle harpoon heads from the Aleutian Islands. Fieldiana, Anthropology, 36(2), 15-23.

Quiroz, D. (2015). Balleneros en la niebla: una mirada paraetnográfica de la caza de ballenas en Chile. Chungara. Revista de Antropología Chilena, 47(2), 319-330.

Ramseyer, D. (1988). Les harpons néolithiques d'Europe occidentale. Bulletin de la Société Préhistorique Française, 85(4), 115-122.

Read, C. (1890). On antiquities from Huasco (Guasco), Chili. The Journal of the Anthropological Institute of Grain Britain and Ireland, 19, 57-63.

Read, C. (1892). An account of a Collection of Ethnographical Specimens formed during Vancouver's voyage in the Pacific Ocean, 1790-1795. The Journal of the Anthropological Institute of Great Britain and Ireland, 21, 99-108.

Read, D. (2007). Artifact classification. A conceptual and methodological approach. California: Left Coast Press.

Rebolledo, S. (2014). Arcaico Medio en la costa arreica: estrategias de caza y pesca costero-maritima en el sitio Zapatero. Memoria para optar al título de Arqueóloga, Universidad de Chile, Santiago.

Rebolledo, S., Béarez, P., Salazar, D. y Fuentes, F. (2015). Maritime fishing during the Middle Holocene in the hyperarid coast of the Atacama Desert. Quaternary International. http: //dx.doi.org/10.1016/j.quaint.2015.09.051
Reeves, R. (2002). The origins and character of 'aboriginal subsistence' whaling: a global review. Mammal Rev., 32(2), 71-106.

Renker, A. (1997). Whale hunting and the Makah tribe: a needs statement. International Whaling Commission Reports, IWC/49/AS5.

Rick, T. y Erlandson, J. (2002). Evaluating the economic significance of sharks, skates, and rays (Elasmobranchs) in prehistoric economies. Journal of Archaeological Science, 29, 111-122.

Rivera, M. y Zlatar, V. (1985). Las estólicas en el desarrollo cultural temprano prehispánico del norte de Chile. En Actas del IX Congreso Nacional de Arqueología Chilena (pp. 14-59). La Serena: Museo Arqueológico de La Serena.

Salazar, D., Castro, V., Michelow, J., Salinas, H., Figueroa, V. y Mille, B. (2010). Minería y metalurgia en la costa arreica de la región de Antofagasta, norte de Chile. Boletín del Museo Chileno de Arte Precolombino, 15(1), 9-23.

Salazar, D., Andrade, P., Borie, C., Escobar, M., Figueroa, V., Flores, C., Olguín, L. y Salinas, H. (2013). Nuevos sitios correspondientes al complejo cultural Huentelauquén en la costa de Taltal. Taltalia, 5, 9-19.

Salazar, D., Figueroa, V., Andrade, P., Salinas, H., Power, X., Rebolledo, S., Parra, S., Orellana, H. y Urrea, J. (2015). Cronología y organización económica de las poblaciones arcaicas de la costa de Taltal. Estudios Atacameños, Arqueologia y Antropología Surandinas, 50, 7-46.

Sayago, C. (1973 [1874]). Historia de Copiapó. Santiago: Editorial Francisco de Aguirre.

Schiappacasse, V. y Niemeyer, H. (1984). Descripción y análisis interpretativo de un sitio Arcaico Temprano en la Quebrada de Camarones. Publicación Ocasional del Museo Nacional de Historia Natural, 41, 1-187.

Sénéchal de la Grange, E. (1903). Pointes de flèches provenantes de la baie d'Antofagasta (Chili). L'homme Préhistorique, 1(6), 161-165.

Silva, J. y Bahamondes, R. (1968). La potera, anzuelo para cefalópodos. Anales del Museo de Historia Natural de Valparaiso, 1, 217-237. 
Silva, J. y Bahamondes, R. (1969). Investigaciones arqueológicas en Taltal. Informe preliminar. Rehue, 2, 7-25.

Skinner, H. (1937). Maori use of the harpoon. The Journal of the Polynesian Society, 46(2), 63-73.

Spahni, J. (1967). Recherches archaeologiques à l'embouchure du rio Loa (Côte du Pacifique-Chili). Journal de la Société des Americanistes, 56(1), 181-239.

Spielmann, K. (2002). Feasting, craft specialization, and the ritual mode of production in small-scale societies. American Anthropologist, 104(1), 195-207.

Stordeur, D. (1980). Typologie et techniques de fabrication des harpons paléoesquimaux de la région d'Igloulik (Canada). Bulletin de la Société Préhistorique Française, 77(8), 239-244.

Stuiver, M., Reimer, P. y Reimer, R. (2005). CALIB 5.0. [www program and documentation]. http: //intcal.qub. ac.uk/calib/manual/index.

Testart, A. (1988). Appropriation of the social product and production relations in hunter-gatherer societies. Dialectical Anthropology, 12(2), 147-164.

True, D. (1975). Early maritime cultural orientations in prehistoric Chile. En Casteel, R. y Quimby, G. (Eds.). Maritime adaptations of the Pacific (pp. 89-143). Paris: Mouton Publishers.

Turner, L. (1889-1890). Ethnology of the Ungava District, Hudson Bay territory. Annual Report of the Bureau of Ethnology of the Secretary of the Smithsonian Institution, 11, 159-350.

Uhle, M. (1917). Los aborigenes de Arica. Santiago: Imprenta Universitaria.

Uhle, M. (1922). Fundamentos étnicos y arqueología de Arica y Tacna. Quito: Imprenta de la Universidad Central.

Vaux, W. (1854). The world encompassed by Sir Francis Drake: being his next voyage to that to Nombre de Dios; collated with an unpublished manuscript of Francis Fletcher, chaplain to the expedition. London: Printed for The Hakluyt Society.

Vázquez de Espinoza, A. (1948 [1630]). Compendio y descripción de las indias occidentales. Washington D.C.: Smithsonian Institution.
Waterman, T. (1920). The whaling equipment of the Makah Indians. University of Washington Publications in Political and Social Science, 1(1), 1-67.

Weniger, G. (1992). Function and form: an ethnoarchaeological analysis of barbed points from northern huntergatherers. En Audouze, F. (Ed.). Ethnoarchaéologie: justification, problèmes, limites (pp. 257-268). Rencontres Internationales d'Archaeologie et d'Histoire d'Antibes 12. Luan-les-Pins: Éditions APDCA.

Weniger, G. (2000). Magdalenian barbed points: harpoons, spears and arrowheads. Anthropologie et Préhistoire, 111, 79-87.

Wintemberg, W. (1906). Bone and horn harpoon heads of Ontario Indians. Annual Archaeological Report for Ontario, 33-56.

Wissler, C. (1916). Harpoons and darts in the Stefansson collection. Anthropological Papers of the American Museum of Natural History, 14(2), 397-475.

Yama'ura, K. (1984). Toggle harpoon heads from Kurigitavik Alaska. Bulletin of the Department of Archaeology of the University of Tokyo, 3, 213-262.

Yellen, J. (1998). Barbed bone points: tradition and continuity in Saharan and Sub-Saharan Africa. The African Archaeological Review, 15(3), 173-198.

Zlatar, V. (1983). Replanteamiento sobre el problema Caleta Huelén 42. Chungara, 10, 21-28.

Zlatar, V. (1989). Un yacimiento precerámico y su problemática desde la perspectiva de sus recintos habitacionales. Hombre y Desierto, 1, 1-36.

Zlatar, V. Ms. s/f. Estructuras habitacionales del sitio Caleta Huelén 42. Cuadernos de campo de excavación. Manuscrito en poder del autor. 